QUARTERLY OF APPLIED MATHEMATICS

VOLUME LXVIII, NUMBER 2

JUNE 2010, PAGES 179-212

S 0033-569X(10)01160-0

Article electronically published on February 17, 2010

\title{
ON PARTIAL SPHERICAL MEANS FORMULAS AND RADIATION BOUNDARY CONDITIONS FOR THE 3+1 WAVE EQUATION
}

\author{
BY
}

STEPHEN R. LAU

Division of Applied Mathematics, Brown University, Providence, Rhode Island 02912, and Department of Mathematics and Statistics, University of New Mexico, Albuquerque, New Mexico 87131

\begin{abstract}
We derive various partial spherical means formulas for the $3+1$ wave equation. Such formulas, considered earlier by both Weston and Teng, involve only partial integration over a solid angle in addition to history-dependent boundary terms, and are appropriate for faces, edges, and corners of "computational domains". For example, a hemispherical means formula corresponds to a face (plane boundary). Exploiting the theory of wave front sets for linear operators developed by Hörmander, Warchall has proved theorems which suggest the existence of "one-sided update formulas" for wave equations. We attempt to realize such an update formula via an explicit construction based on our hemispherical means formula. We focus on face points and plane boundaries, but also introduce one-fourth and one-eighth spherical means formulas with most of our arguments going through for a point located on either a domain edge or a corner. Throughout our analysis we encounter a number of, we believe, heretofore unknown identities for classical solutions to the wave equation.
\end{abstract}

1. Introduction. Specification and efficient numerical implementation of exact radiation boundary conditions (RBC) for slender or complicated domains, possibly with sharp corners, is a fundamental problem in computational mathematics with broad applications in the sciences. Even for the wave equation, the problem is essentially open for domains which are slender or otherwise complicated by edges and corners, although this view is subject to revision in light of recent breakthroughs [1, 2] on "corner compatibility conditions" by Hagstrom, Warburton, and Givoli. Successful implementations of RBC do exist for boundaries which are highly symmetric, such as infinite planes, spheres, and infinite cylinders. In particular, Alpert, Greengard, and Hagstrom (AGH) described an implementation based on Laplace convolution for the $3+1$ wave equation

Received January 4, 2008.

2000 Mathematics Subject Classification. Primary 35L05, 78A40.

Supported through grants ARO DAAD19-03-1-0146 (to UNM) and DMS 0554377 and DARPA/AFOSR FA9550-05-1-0108 (both to Brown University).

E-mail address: lau@dam.brown.edu 


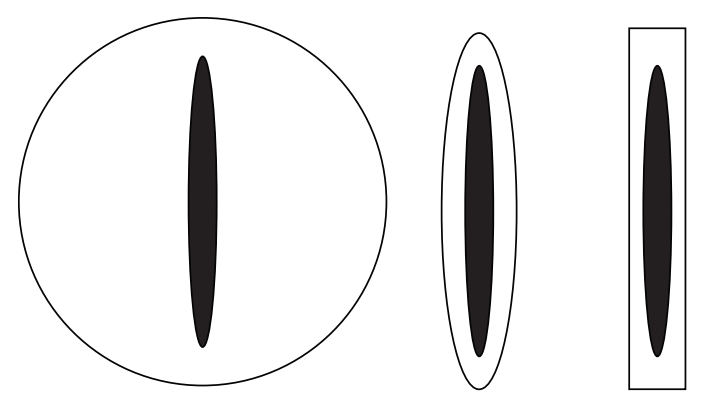

Fig. 1. Idealized Representation of a SCATter. The solid ellipsoid represents a high aspect ratio scatterer within a computational domain determined by a bounding surface. A spherical boundary is depicted on the left. The two boundaries depicted on the right are adapted to the geometry, affording smaller computational domains.

[3. The AGH approach has been extended both to plane and cylindrical boundaries [4] and to a number of other equations. For example, it has been successfully applied to both the Schrödinger equation by Jiang [5] and the linear PDE describing perturbations of Schwarzschild black holes [6, 7, 8]. In each case, a spherical boundary is assumed. However, despite the theoretical and practical importance of this approach, the requirement of a spherical boundary, or otherwise highly symmetric boundary, is often a severe limitation. Indeed, in modeling wave phenomena involving slender geometries, say the scattering of acoustic waves off of a submarine, the choice of a spherical boundary inevitably wastes computational zones, as suggested in Figure 1.

Exploiting the theory [9, 10] of wave front sets for linear operators developed by Hörmander, Warchall has proved theorems suggesting the existence of "one-sided update formulas" for wave equations [11. For classical solutions to the $3+1$ wave equation $\left(-\partial_{t}^{2}+\Delta_{\mathbf{x}}\right) U=0$, the ramifications of his work are described as follows (see Theorem 6 of [11] for details). Consider three times $t_{0}<t_{1}<t_{2}=T$, a spatial point $\mathbf{X}$ on the boundary $\partial \Sigma$ of an open convex set $\Sigma$, and $\mathcal{O}=\Sigma \cap\left\{\mathbf{x}:|\mathbf{x}-\mathbf{X}| \leq T-t_{1}\right\}$. If (i) the data $\left\{U\left(t_{1}, \mathbf{x}\right), U_{t}\left(t_{1}, \mathbf{x}\right)\right\}$ vanishes on an open set that contains $\mathcal{O}$ and (ii) the data $\left\{U\left(t_{0}, \mathbf{x}\right), U_{t}\left(t_{0}, \mathbf{x}\right)\right\}$ is supported on the interior of $\Sigma$, then $U(T, \mathbf{X})=0$. Notice that proviso (ii) restricts the $t_{0}$-data to the "computational domain" in the (perhaps ancient) past. By uniqueness of solutions to the wave equation, we may then conclude that for such $t_{0}$-data the value $U(T, \mathbf{X})$ is determined solely by the $t_{1}$-data on $\mathcal{O}$. Through the introduction of a conjectured one-sided update formula, Warchall has suggested that this result might yield a practical way of numerically implementing RBC for the wave equation.

In this paper we attempt to realize Warchall's conception for the $3+1$ wave equation via explicit constructions, although truly succeeding only for a limited class of solutions. Various partial spherical means formulas serve as the starting point for our analysis. Such formulas involve only partial integration over a solid angle in addition to historydependent boundary terms, and are appropriate for faces, edges, and corners of "computational domains." These formulas are essentially the same as those studied by Teng 
[12] and also exhaustively for a plane boundary by Weston [13. We use these formulas to obtain new split level formulas which also depend on the intermediate time $t_{1}$, and this is a key difference between our work and theirs. At least within the spacetime neighborhoods in which our analysis takes place, we work with classical solutions to the homogeneous $3+1$ wave equation. While our assumptions are indeed more restrictive than the setting adopted by Warchall, they are reasonable from the standpoint of numerical computations. While our motivation stems solely from numerical applications, this is essentially a theoretical paper, chiefly because we do not yet know if our current results suggest a practical numerical implementation. Therefore, one might reasonably view this as a failed work. Nevertheless, our main results are the following.

- For domain faces, edges, and corners, we introduce partial spherical means formulas with history terms. Some of these formulas are the same as those considered by Weston [13] and Teng [12, but others are slightly more general (either in terms of geometry or in featuring multiple times).

- We express the history terms arising in partial spherical means formulas solely in terms of the intermediate time level $t_{1}$, thereby achieving split level formulas which express $U(T, \mathbf{X})$ solely in terms of the data at two initial times, $t_{0}$ and $t_{1}$. Assuming appropriate initial data at time $t_{0}$, we then formally quasi-localize in space (to the backward lightcone of $(T, \mathbf{X})$ ) the already time-localized new expressions for the history terms, thereby nearly realizing Warchall's conception for sufficiently general solutions. We explain why our construction falls short.

- We give a constructive proof of Warchall's theorem for a certain class of pointsource solutions to the $3+1$ wave equation. From the proof one could write down an explicit update formula (i.e., realize Warchall's conception for such solutions), although we are unsure of its utility for a numerical implementation.

In addition, we offer what we see as the key obstacles to realizing Warchall's conception in a sufficiently general setting.

The organization of this paper is as follows. In the next subsection we prove a lemma establishing partial spherical means formulas and describe some concrete examples, including several hemispherical means formulas. Section 3 focuses on a split level formula for plane boundaries, our attempt at a concrete realization of Warchall's conception for sufficiently general solutions. Section 4 gives the derivation of the split level formula. For a general multipole solution to the wave equation, Section 5 presents a concrete demonstration of Warchall's conception. Section 6 considers edges and corners, and the extent to which our results generalize to these scenarios. We include a number of appendices. The first shows that our hemispherical means formula is equivalent to the AGH

plane boundary conditions based on the Laplace convolution technique. The remaining appendices collect further technical details not presented in the text.

\section{Preliminaries.}

2.1. Notation. Consider Minkowski spacetime, that is, $\mathbb{R}^{4}$ equipped with the pseudoRiemannian line-element

$$
\mathrm{d} s^{2}=-\mathrm{d} t^{2}+\mathrm{d} r^{2}+r^{2}\left(\mathrm{~d} \theta^{2}+\sin ^{2} \theta \mathrm{d} \phi^{2}\right),
$$


here written in time spherical polar coordinates. The factor $\mathrm{d} \theta^{2}+\sin ^{2} \theta \mathrm{d} \phi^{2}$ is the lineelement of a unit-radius round sphere $S^{2}$, which has $1 \mathrm{~d} \Omega=\mathrm{d} \phi \mathrm{d} \theta \sin \theta$ as its area element. Consider a spacetime point $p$ with Cartesian coordinates $(T, X, Y, Z)$, assuming $T>0$. With $\mathbf{x}=(x, y, z), \mathbf{X}=(X, Y, Z)$, and $r=|\mathbf{x}-\mathbf{X}|$, the past null cone of $p$ is the locus of points obeying $t+r=T$. Denote by $B_{\mathbf{X}}(t, r)$ the radius $-r$ round sphere at time $t$ and centered at the spatial point $\mathbf{X}$. The center of $B_{\mathbf{X}}(t, r)$ is not $p$ unless $t=T$. $B_{\mathbf{X}}(t, T-t)$ is the intersection of time level $t$ with the past null cone of $p$. When considering a hemispherical means formula below, we split $B_{\mathbf{X}}(t, T-t)$ into $B_{\mathbf{X}}^{+}(t, T-t)$ and $B_{\mathbf{X}}^{-}(t, T-t)$, according to whether $z>Z$ or $z<Z$. We also define the circular ring $\Re_{\mathbf{X}}(t, T-t)=\operatorname{cl} B_{\mathbf{X}}^{+}(t, T-t) \cap \operatorname{cl} B_{\mathbf{X}}^{-}(t, T-t)$, the intersection of the boundary plane $z=Z$ at time level $t$ with the past null cone of $p$. We view $B_{\mathbf{X}}^{-}(t, T-t)$ as inside the computational domain, and $B_{\mathbf{X}}^{+}(t, T-t)$ as outside. When $\mathbf{X}$ is the origin $\mathbf{0}$, we simply write, for example, $B(t, T-t)$ and $\Re(t, T-t)$, rather than $B_{\mathbf{0}}(t, T-t)$ and $\Re_{\mathbf{0}}(t, T-t)$.

Let $\mathcal{S}^{*}$ be the angular parameter space specifying a spherical polygon, that is, a portion of the unit sphere enclosed by the arcs of great circles. Let $B^{*}(t, r)$ be a corresponding spherical polygon contained in $B(t, r)$. The boundary $\partial B^{*}(t, r)$ of $B^{*}(t, r)$ is a closed, continuous, and piecewise smooth curve $\gamma(t, r)$, and it may be a single great circle. Expressing the boundary $\gamma(t, r)$ as a union $\bigcup_{i} \gamma_{i}(t, r)$ of smooth curves, we use $\mathrm{d} \sigma_{i}=$ $\mathrm{d} \phi_{i} r$ to represent the induced Riemannian measure (differential of arc-length) on the component $\gamma_{i}(t, r)$, where $\phi_{i}$ is an angular coordinate along the component. Furthermore, $\partial / \partial \mathrm{x}^{i}$ will represent the Cartesian direction which coincides on $\gamma_{i}(t, r)$ with the curves's outward-pointing normal as a component of $\partial B^{*}(t, r)$. The $\partial / \partial \mathrm{x}^{i}$ direction need not be one of the fixed Cartesian basis directions $\partial / \partial x, \partial / \partial y, \partial / \partial z$. Along $\gamma_{i}(t, r)$ the vector field $\partial / \partial \mathrm{x}^{i}$ points perpendicularly to $\gamma_{i}(t, r)$ and also tangent to $B(t, r)$.

For $W=W(t, x, y, z)$ we introduce the following convention for (unnormalized) angular averages:

$$
\langle W\rangle_{B^{*}(t, r)}=\int_{\mathcal{S}^{*}} \mathrm{~d} \Omega W(t, r \sin \theta \cos \phi, r \sin \theta \sin \phi, r \cos \theta),
$$

where $B^{*}(t, r)$ is the radius- $r$ spherical portion centered at the origin for which $(\theta, \phi) \in$ $\mathcal{S}^{*}$. This average does not use the proper area measure $\mathrm{d} \Omega r^{2}$ on $B^{*}(t, r)$. By choosing not to incorporate the proper area measure in the definition of the average, we both slightly streamline the appearance of many formulas and ensure that $r \rightarrow 0^{+}$limits are readily taken. We will write

$$
\langle W\rangle_{B_{\mathbf{X}}(t, r)}=\int_{S^{2}} \mathrm{~d} \Omega W(t, X+r \sin \theta \cos \phi, Y+r \sin \theta \sin \phi, Z+r \cos \theta)
$$

when the average (in this example over a whole sphere) is taken about a generic spatial point $\mathbf{X}$. Furthermore, we write

$$
[W]_{\Re \mathbf{x}(t, \rho)}=\int_{0}^{2 \pi} \mathrm{d} \phi W(t, X+\rho \cos \phi, Y+\rho \sin \phi, Z)
$$

for ring averaging based at $\mathbf{X}$.

\footnotetext{
${ }^{1}$ Because we will encounter many long integral expressions, we find it useful to use the physicists' convention of putting all coordinate measures to the left.
} 
2.2. Partial spherical means formulas. To derive these, first let $\mathcal{M}=\{(t, \mathbf{x}): \mathbf{x} \leq$ $T-t\}$ represent the solid past null cone of the spacetime point $(T, 0,0,0)$. For a generic time $t<T$, let $\mathcal{M}_{t}$ represent the closed portion of $\mathcal{M}$ lying to the future of time level $t$. Partial spherical means formulas arise from the following (also given in [14]).

LEMma 2.1. Let $U$ be a classical solution to the wave equation on a neighborhood of $\mathcal{M}_{t}$. Then, with $q$ the solid angle subtended by the spherical polygon $B^{*}(t, r)$, we have

$$
\begin{aligned}
q U(T, 0,0,0)= & \sum_{i} \int_{t}^{T} \mathrm{~d} \tau \frac{1}{T-\tau} \int_{\gamma_{i}(\tau, T-\tau)} \mathrm{d} \sigma_{i} \frac{\partial U}{\partial x^{i}} \\
& (T-t)\left\langle U_{t}\right\rangle_{B^{*}(t, T-t)}+\partial_{T}(T-t)\langle U\rangle_{B^{*}(t, T-t)} .
\end{aligned}
$$

The result can be shifted to a generic spatial point $\mathbf{X}$ by translation invariance.

Proof. We first note that the equation holds in the $t \rightarrow T^{-}$limit. Indeed, in each integral over $\gamma_{i}(\tau, T-\tau)$ the apparently singular $(T-\tau)^{-1}$ is canceled by a $(T-\tau)$ in the $\mathrm{d} \sigma_{i}$ measure. Therefore, to gather the result, we must simply establish that the right-hand side of (2.5) is constant in $t$. With that aim, we consider the following key identity:

$$
\begin{aligned}
(T-t)^{-1} & \Delta U(t,(T-t) \boldsymbol{\nu}) \\
& =\partial_{t}\left\{(T-t) U_{t}(t,(T-t) \boldsymbol{\nu})+\partial_{T}[(T-t) U(t,(T-t) \boldsymbol{\nu})]\right\},
\end{aligned}
$$

where $\Delta$ is the $S^{2}$ Laplacian and $\boldsymbol{\nu}$ a set of direction cosines. This identity is the wave equation expressed in spherical polar coordinates. Indeed, note that the left-hand side of (2.6) is symbolically

$$
\left(\partial_{t}-\partial_{R}\right) R\left(\mathrm{U}_{t}+\mathrm{U}_{R}+\mathrm{U} / R\right)=R\left(\mathrm{U}_{t t}-\mathrm{U}_{R R}-2 \mathrm{U}_{R} / R\right) .
$$

Since the angular parameter space $\mathcal{S}^{*}$ does not depend on time, we may integrate (2.6), thereby obtaining

$$
\begin{aligned}
(T-t)^{-1} & \int_{\mathcal{S}^{*}} \mathrm{~d} \Omega \Delta U(t,(T-t) \boldsymbol{\nu}) \\
& =\partial_{t} \int_{\mathcal{S}^{*}} \mathrm{~d} \Omega\left\{(T-t) U_{t}(t,(T-t) \boldsymbol{\nu})+\partial_{T}[(T-t) U(t,(T-t) \boldsymbol{\nu})]\right\},
\end{aligned}
$$

or in our more compact notation,

$$
(T-t)^{-1}\langle\Delta U\rangle_{B^{*}(t, T-t)}=\partial_{t}(T-t)\left\langle U_{t}\right\rangle_{B^{*}(t, T-t)}+\partial_{t} \partial_{T}(T-t)\langle U\rangle_{B^{*}(t, T-t)} .
$$

By Stokes' Theorem, the term on the left-hand side of the equation integrates to

$$
(T-t)^{-1}\langle\Delta U\rangle_{B^{*}(t, T-t)}=\sum_{i} \frac{1}{T-t} \int_{\gamma_{i}(t, T-t)} \mathrm{d} \sigma_{i} \frac{\partial U}{\partial \mathrm{x}_{i}},
$$

that is, precisely minus the time derivative of the first term on the right-hand side of (2.5), whence the right-hand side of (2.5) is indeed constant in $t$.

The lemma can also be proved with distributions or by generalizing Hadamard's approach for deriving the spherical means formula [15, 16. We have carried out Hadamard's approach for several partial spherical means formulas. This route may prove a useful tool 
when the angular parameter space is time-dependent. When $B^{*}(t, T-t)$ is the entire sphere $B(t, T-t)$, the lemma (2.1) yields the standard spherical means formula [17, 18]

$$
4 \pi U(T, 0,0,0)=(T-t) \int_{S^{2}} \mathrm{~d} \Omega U_{t}(t,(T-t) \boldsymbol{\nu})+\frac{\partial}{\partial T}\left[(T-t) \int_{S^{2}} \mathrm{~d} \Omega U(t,(T-t) \boldsymbol{\nu})\right] .
$$

In a sense, the spherical means formula holds because the "boundary of a boundary is zero" [19]. When $\gamma(t, T-t)$ is the equatorial great circle lying in the plane $z=Z$, the lemma yields

$$
\begin{aligned}
& 2 \pi U(T, 0,0,0)=-\int_{t}^{T} \mathrm{~d} \tau \int_{0}^{2 \pi} \mathrm{d} \phi U_{z}(\tau,(T-\tau) \cos \phi,(T-\tau) \sin \phi, 0) \\
& \quad+(T-t) \int_{\mathcal{S}^{+}} \mathrm{d} \Omega U_{t}(t,(T-t) \boldsymbol{\nu})+\frac{\partial}{\partial T}\left[(T-t) \int_{\mathcal{S}^{+}} \mathrm{d} \Omega U(t,(T-t) \boldsymbol{\nu})\right]
\end{aligned}
$$

with $\mathcal{S}^{+}=\{(\theta, \phi): 0 \leq \theta<\pi / 2,0 \leq \phi<2 \pi\}$ representing the angular parameter space specifying the northern hemisphere. $\mathcal{S}^{-}$specifies the southern hemisphere. Edge and corner examples of the lemma are given in Section 6 .

2.3. Hemispherical means formulas. This subsection elaborates on the hemispherical example (2.12) . Formulas given in 2.3.1 can be generalized to edge and corner formulas, while some of those in 2.3 .2 are specific to a plane boundary.

2.3.1. Consequences of the formula. We first use our notation for angular averages to express (2.12) in the following succinct form:

$$
\begin{aligned}
2 \pi U(T, 0,0,0)= & -\int_{t}^{T} \mathrm{~d} \tau\left[U_{z}\right]_{\Re(\tau, T-\tau)} \\
& +(T-t)\left\langle U_{t}\right\rangle_{B^{+}(t, T-t)}+\partial_{T}(T-t)\langle U\rangle_{B^{+}(t, T-t)} .
\end{aligned}
$$

Combining (2.13) with

$$
4 \pi U(T, 0,0,0)=(T-t)\left\langle U_{t}\right\rangle_{B(t, T-t)}+\partial_{T}(T-t)\langle U\rangle_{B(t, T-t)}
$$

that is, the ordinary spherical means formula (2.11), we obtain the mirror formula of (2.13),

$$
\begin{aligned}
2 \pi U(T, 0,0,0)= & \int_{t}^{T} \mathrm{~d} \tau\left[U_{z}\right]_{\Re(\tau, T-\tau)} \\
& +(T-t)\left\langle U_{t}\right\rangle_{B^{-}(t, T-t)}+\partial_{T}(T-t)\langle U\rangle_{B^{-}(t, T-t)} .
\end{aligned}
$$

As with (2.13), or indeed the ordinary spherical means formula, (2.15) requires no special assumptions on the support of the initial data.

To achieve more interesting consequences of our result, we suppose that the initial data vanishes for $z>-\delta$ (with $\delta>0$ small). More precisely, it is enough to assume that the data vanishes on a neighborhood of $\mathrm{cl} B^{+}\left(t_{0}, T-t_{0}\right)$. Then we may choose $t=t_{0}$ in 
(2.13) to find 2

$$
2 \pi U(T, 0,0,0) \stackrel{\text { CID }}{=}-\int_{t_{0}}^{T} \mathrm{~d} \tau\left[U_{z}\right]_{\Re(\tau, T-\tau)} .
$$

The result (2.16) was obtained and exploited by Weston [13] and also appears in the monograph [17]. Next, we replace $t$ with $t_{1}$ in (2.15), assuming $t_{0}<t_{1}<T$, and add the result to (2.16), thereby reaching

$$
\begin{aligned}
4 \pi U(T, 0,0,0) \stackrel{\mathrm{CID}}{=} & -\int_{t_{0}}^{t_{1}} \mathrm{~d} \tau\left[U_{z}\right]_{\Re(\tau, T-\tau)} \\
& +\left(T-t_{1}\right)\left\langle U_{t}\right\rangle_{B^{-}\left(t_{1}, T-t_{1}\right)}+\partial_{T}\left(T-t_{1}\right)\langle U\rangle_{B^{-}\left(t_{1}, T-t_{1}\right)} .
\end{aligned}
$$

We view this as the key formula of this section. Replacing $t_{1}$ with $t_{0}$ in (2.17), we get

$$
4 \pi U(T, 0,0,0) \stackrel{\mathrm{CID}}{=}\left(T-t_{0}\right)\left\langle U_{t}\right\rangle_{B^{-}\left(t_{0}, T-t_{0}\right)}+\partial_{T}\left(T-t_{0}\right)\langle U\rangle_{B^{-}\left(t_{0}, T-t_{0}\right)},
$$

that is, the standard spherical means formula applied to our special initial data at $t_{0}$.

2.3.2. Other versions of the hemispherical means formula. Since $U_{z}$ solves the wave equation if $U$ does (assuming requisite smoothness), from (2.17) we hav 3

$$
\begin{aligned}
4 \pi U_{z}(T, 0,0,0) \stackrel{\mathrm{CID}}{=} & -\int_{t_{0}}^{t_{1}} \mathrm{~d} \tau\left[U_{z z}\right]_{\Re(\tau, T-\tau)} \\
& +\left(T-t_{1}\right)\left\langle U_{t z}\right\rangle_{B^{-}\left(t_{1}, T-t_{1}\right)}+\partial_{T}\left(T-t_{1}\right)\left\langle U_{z}\right\rangle_{B^{-}\left(t_{1}, T-t_{1}\right)} .
\end{aligned}
$$

Written in cylindrical coordinates, the wave equation is

$$
U_{t t}-U_{z z}=U_{x x}+U_{y y}=\rho^{-1}\left(\rho U_{\rho}\right)_{\rho}+\rho^{-2} U_{\phi \phi},
$$

where $\rho=\sqrt{x^{2}+y^{2}}$. Writing (2.20) as

$$
U_{z z}=\left(\partial_{t}-\partial_{\rho}\right)\left(U_{t}+U_{\rho}\right)-\rho^{-1} U_{\rho}-\rho^{-2} U_{\phi \phi}
$$

and substituting the right-hand side into (2.19), we obtain

$$
\begin{aligned}
4 \pi U_{z}(T, 0,0,0) \stackrel{\mathrm{CID}}{=} & \int_{t_{0}}^{t_{1}} \mathrm{~d} \tau \frac{1}{T-\tau} \partial_{T}[U]_{\Re(\tau, T-\tau)}-\left(\partial_{t_{1}}+2 \partial_{T}\right)[U]_{\Re\left(t_{1}, T-t_{1}\right)} \\
& +\left(T-t_{1}\right)\left\langle U_{t z}\right\rangle_{B^{-}\left(t_{1}, T-t_{1}\right)}+\partial_{T}\left(T-t_{1}\right)\left\langle U_{z}\right\rangle_{B^{-}\left(t_{1}, T-t_{1}\right)}
\end{aligned}
$$

To reach this formula, we have used the facts that $\partial / \partial t-\partial / \partial \rho$ and $\partial / \partial \rho$ respectively correspond to $\partial / \partial \tau$ and $\partial / \partial T$, and also

$$
-\left(\partial_{t_{1}}+2 \partial_{T}\right)[U]_{\Re\left(t_{1}, T-t_{1}\right)}=-\left[U_{t}+U_{\rho}\right]_{\Re\left(t_{1}, T-t_{1}\right)} .
$$

2 The acronym CID stands for computational initial data, that is, initial data at time $t_{0}$ which is supported on the interior of the computational domain, whence $P \stackrel{\text { CID }}{=} Q$ is a true equality provided we assume computational initial data. In many, but not all, of the formulas which follow, one may alternatively interpret $P \stackrel{\text { CID }}{=} Q$ as shorthand for

$$
P=Q+\left(T-t_{0}\right)\left\langle U_{t}\right\rangle_{B_{\mathbf{X}}^{+}\left(t_{0}, T-t_{0}\right)}+\partial_{T}\left(T-t_{0}\right)\langle U\rangle_{B_{\mathbf{X}}^{+}\left(t_{0}, T-t_{0}\right)},
$$

where in this subsection $\mathbf{X}=(0,0,0)$, but later on $\mathbf{X}$ will be generic. Alternative interpretations for $P \stackrel{\text { CID }}{=} Q$ are more complicated for some formulas in Sections 2.3 .2 and 3 and for the edge and corner scenarios in Section 6 We also refer to initial data which "obeys the CID condition" (or a similar phrase). In that case, we do assume computational initial data, with no alternative interpretation possible.

${ }^{3}$ The author thanks T. Hagstrom for the following argument. 
The $t_{1} \rightarrow T^{-}$limit of (2.22) then yields

$$
U_{t}(T, 0,0,0)+U_{z}(T, 0,0,0) \stackrel{\mathrm{CID}}{=} \frac{1}{2 \pi} \int_{t_{0}}^{T} \mathrm{~d} \tau \frac{1}{(T-\tau)} \partial_{T}[U]_{\Re(\tau, T-\tau)},
$$

since the hemispherical means terms in (2.22) tend to $2 \pi U_{z}(T, 0,0,0)$ as $t_{1} \rightarrow T^{-}$. Moreover, since $U_{\rho}$ will average to zero in the limit, the integral $-\left[U_{t}+U_{\rho}\right]_{\Re\left(t_{1}, T-t_{1}\right)}$ as a whole tends to $-2 \pi U_{t}(T, 0,0,0)$. Weston also found the formula (2.24) in his "splitting" analysis of the wave equation [13. Appendix $\mathrm{A}$ shows that (2.24) is the physicalspace version of a plane boundary condition derived in Ref. 4. Other expressions for $U_{t}(T, 0,0,0)+U_{z}(T, 0,0,0)$ are possible. To get one, we replace $U$ by $U_{t}$ in (2.17), whereupon finding after an integration by parts that

$$
\begin{aligned}
4 \pi U_{t}(T, 0,0,0) \stackrel{\mathrm{CID}}{=} & -\int_{t_{0}}^{t_{1}} \mathrm{~d} \tau \partial_{T}\left[U_{z}\right]_{\Re(\tau, T-\tau)}-\left[U_{z}\right]_{\Re\left(t_{1}, T-t_{1}\right)} \\
& +\left(T-t_{1}\right)\left\langle U_{t t}\right\rangle_{B^{-}\left(t_{1}, T-t_{1}\right)}+\partial_{T}\left(T-t_{1}\right)\left\langle U_{t}\right\rangle_{B^{-}\left(t_{1}, T-t_{1}\right)} .
\end{aligned}
$$

This formula is closely related to the one obtained via a straightforward differentiation of (2.17) by $\partial / \partial T$, with the two related by an angular integration by parts at time level $t_{1}$. We find (another Weston formula)

$$
U_{t}(T, 0,0,0)+U_{z}(T, 0,0,0) \stackrel{\mathrm{CID}}{=}-\frac{1}{2 \pi} \int_{t_{0}}^{T} \mathrm{~d} \tau \partial_{T}\left[U_{z}\right]_{\Re(\tau, T-\tau)},
$$

after taking the $t_{1} \rightarrow T^{-}$limit in (2.25).

3. Split level initial value problem. Using translation invariance and restoring the integrals at time $t_{0}$, we may express (2.17) as follows:

$$
\begin{aligned}
4 \pi U(T, \mathbf{X}) & =\left(T-t_{0}\right)\left\langle U_{t}\right\rangle_{B_{\mathbf{X}}^{+}\left(t_{0}, T-t_{0}\right)}+\partial_{T}\left(T-t_{0}\right)\langle U\rangle_{B_{\mathbf{X}}^{+}\left(t_{0}, T-t_{0}\right)} \\
& +\left(T-t_{1}\right)\left\langle U_{t}\right\rangle_{B_{\mathbf{X}}^{-}\left(t_{1}, T-t_{1}\right)}+\partial_{T}\left(T-t_{1}\right)\langle U\rangle_{B_{\mathbf{X}}^{-}\left(t_{1}, T-t_{1}\right)}+\mathcal{H}\left(t_{0}, t_{1}\right),
\end{aligned}
$$

where 4

$$
\mathcal{H}\left(t_{0}, t_{1}\right) \equiv-\int_{t_{0}}^{t_{1}} \mathrm{~d} \tau \int_{0}^{2 \pi} \mathrm{d} \phi U_{z}(\tau, X+(T-\tau) \cos \phi, Y+(T-\tau) \sin \phi, Z) .
$$

This section describes an alternative formula for the history term $\mathcal{H}\left(t_{0}, t_{1}\right)$ in (3.1), one which depends on data solely at time level $t_{1}$. We therefore arrive at a formula expressing the value $U(T, \mathbf{X})$ in terms of data at two initial times, $t_{0}$ and $t_{1}$. One version of this split level formula is our best attempt at a sufficiently general realization of Warchall's conception. For simplicity, this section only considers the plane boundary case, but the results carry over to the edge and corner scenarios described in Section 6 .

\footnotetext{
${ }^{4}$ Assuming the initial data obeys the CID condition (cf. footnote 2), the $t_{0}$ in $\mathcal{H}\left(t_{0}, t_{1}\right)$ may be replaced by any later time $t_{0}+\delta t$, provided that no radiation intersects the ring history $\left\{\Re_{\mathbf{X}}(t, T-t)\right.$ : $\left.t \in\left[t_{0}, t_{0}+\delta t\right]\right\}$. Therefore, under this assumption, $\mathcal{H}\left(t_{0}+\delta, t_{1}\right)=\mathcal{H}\left(t_{0}, t_{1}\right)$. Such insensitivity to $t_{0}$ also holds for other incarnations of $\mathcal{H}\left(t_{0}, t_{1}\right)$ derived below, Eqs. (3.4]3.6]4.15]4.17). In particular, numerical experimentation with (3.6) has confirmed this insensitivity.
} 
3.1. Main formula. To write down the formula, we introduce a parameterization

$$
\mathbf{X}\left(T-t_{0}, \phi\right)=\left(X+\left(T-t_{0}\right) \cos \phi, Y+\left(T-t_{0}\right) \sin \phi, Z\right)
$$

of the ring $\Re_{\mathbf{X}}\left(t_{0}, T-t_{0}\right)$, and then define a radial variable $R=\left|\mathbf{X}\left(T-t_{0}, \phi\right)-\mathbf{x}\right|$. Our split level formula is determined by a new expression for the history term,

$$
\begin{aligned}
\mathcal{H}\left(t_{0}, t_{1}\right) & =\int_{0}^{2 \pi} \mathrm{d} \phi \int_{B_{\mathbf{X}\left(T-t_{0}, \phi\right)}\left(t_{1}, t_{1}-t_{0}\right)} \mathrm{d} S_{\mathbf{x}} \mathcal{K}\left(T-t_{0}, \mathbf{X}-\mathbf{x}, \phi\right) \mathcal{G}\left(t_{0}+R, \mathbf{x}\right), \\
\mathcal{G}\left(t_{0}+R, \mathbf{x}\right) & =\frac{1}{4 \pi R}\left[-U_{t}\left(t_{0}+R, \mathbf{x}\right)+\frac{\partial U}{\partial R}\left(t_{0}+R, \mathbf{x}\right)+\frac{1}{R} U\left(t_{0}+R, \mathbf{x}\right)\right],
\end{aligned}
$$

where the integration is over data only at time $t_{1}$. Indeed, for points on the sphere $B_{\mathbf{X}\left(T-t_{0}, \phi\right)}\left(t_{1}, t_{1}-t_{0}\right)$, we have $\left|\mathbf{X}\left(T-t_{0}, \phi\right)-\mathbf{x}\right|=\left(t_{1}-t_{0}\right)$ so that $t_{0}+R=t_{1}$. The area element $\mathrm{d} S_{\mathbf{x}}=\mathrm{d} \Omega_{\mathbf{x}}\left(t_{1}-t_{0}\right)^{2}$. Also appearing in (3.4) is the kernel

$$
\mathcal{K}\left(T-t_{0}, \mathbf{X}-\mathbf{x}, \phi\right)=\frac{-(Z-z)}{\left(T-t_{0}\right)+(X-x) \cos \phi+(Y-y) \sin \phi-\left|\mathbf{X}\left(T-t_{0}, \phi\right)-\mathbf{x}\right|} .
$$

We note that $\mathcal{K}\left(T-t_{0}, \mathbf{X}-\mathbf{x}, \phi\right)$ depends solely on the difference $\mathbf{X}-\mathbf{x}$ and is harmonic (solves Laplace's equation) in either variable. Moreover, it is annihilated by $\partial / \partial T+\partial / \partial t_{0}$ and satisfies $\nabla_{\mathbf{X}} R \cdot \nabla_{\mathbf{X}} \mathcal{K}\left(T-t_{0}, \mathbf{X}-\mathbf{x}, \phi\right)=0$.

Using the analysis in Section 4, we write down another version of (3.4). Namely,

$$
\mathcal{H}\left(t_{0}, t_{1}\right)=\int_{0}^{2 \pi} \mathrm{d} \phi \int_{S^{2}} \mathrm{~d} \Omega k(\alpha, \beta) g_{\phi}(\alpha, \beta),
$$

where $\mathrm{d} \Omega=\mathrm{d} \beta \mathrm{d} \alpha \sin \alpha$,

$$
\begin{aligned}
g_{\phi}(\alpha, \beta)= & -\frac{\left(t_{1}-t_{0}\right)}{2 \pi} U_{t}\left(t_{1}, \mathbf{X}\left(T-t_{0}, \phi\right)+\left(t_{1}-t_{0}\right) \boldsymbol{\mu}(\phi, \alpha, \beta)\right) \\
& +\frac{\partial}{\partial t_{1}} \frac{\left(t_{1}-t_{0}\right)}{4 \pi} U\left(t_{1}, \mathbf{X}\left(T-t_{0}, \phi\right)+\left(t_{1}-t_{0}\right) \boldsymbol{\mu}(\phi, \alpha, \beta)\right),
\end{aligned}
$$

and the angular kernel $k(\alpha, \beta)$ stemming from $\mathcal{K}\left(T-t_{0}, \mathbf{X}-\mathbf{x}, \phi\right)$ is

$$
k(\alpha, \beta)=\frac{\sin \alpha \cos \beta}{\cos \alpha-1} .
$$

Here $\boldsymbol{\mu}(\phi, \alpha, \beta)$ are certain direction cosines, listed later in (4.7), whose precise form need not concern us now. The described scenario comes from (4.15) in Subsection 4.2,

3.2. Associated Poisson problem. The following is a key observation:

$$
\int_{S^{2}} \mathrm{~d} \Omega g_{\phi}(\alpha, \beta)=U\left(t_{0}, \mathbf{X}\left(T-t_{0}, \phi\right)\right),
$$

because the inner integral in (3.4), with $\mathcal{K}\left(T-t_{0}, \mathbf{X}-\mathbf{x}, \phi\right)$ set to unity, is the future spherical means formula (see Subsection 4.1) for the past value $U\left(t_{0}, \mathbf{X}\left(T-t_{0}, \phi\right)\right)$. Obviously, we then have (cf. footnote 2)

$$
\int_{S^{2}} \mathrm{~d} \Omega g_{\phi}(\alpha, \beta) \stackrel{\mathrm{CID}}{=} 0 .
$$

Therefore, given initial data obeying the CID condition, the function $g_{\phi}(\alpha, \beta)$ satisfies the Neumann compatibility requirement for a solvable Poisson problem on the sphere 
$S^{2}$, and we are guaranteed a solution $u_{\phi}$ to the Poisson problem $\Delta u_{\phi}=g_{\phi}$, which is unique up to an unimportant constant. More generally, we have a $\phi$-dependent average $\widehat{g}_{\phi}$ defined by

$$
4 \pi \widehat{g}_{\phi}=\int_{S^{2}} d \Omega g_{\phi}(\alpha, \beta), \quad 4 \pi \widehat{g}_{\phi}=U\left(t_{0}, \mathbf{X}\left(T-t_{0}, \phi\right)\right),
$$

and the shifted problem $\Delta u_{\phi}=g_{\phi}-\widehat{g}_{\phi}$ is uniquely solvable up to a constant.

At all points, save for the $\alpha=0$ North Pole where it is singular, the kernel $k(\alpha, \beta)$ is annihilated by the spherical Laplacian

$$
\Delta \equiv \frac{\partial^{2}}{\partial \alpha^{2}}+\cot \alpha \frac{\partial}{\partial \alpha}+\csc ^{2} \alpha \frac{\partial^{2}}{\partial \beta^{2}} .
$$

Our expression $-k(\alpha, \beta) / \cos \beta$ is the $m=1$ "fundamental solution" discussed in [20]. It is also closely related to the Green's function for $\Delta$, that is 21

$$
G(\alpha, \beta ; \gamma, \omega)=-\ln \frac{1}{|1-\sin \alpha \cos \beta \sin \gamma \cos \omega-\sin \alpha \sin \beta \sin \gamma \sin \omega-\cos \alpha \cos \gamma|} .
$$

Indeed, taking the $\alpha$ derivative of $G(\alpha, \beta ; \gamma, \omega)$ and then setting $\gamma=0$, we again find $-k(\alpha, \beta) / \cos \beta$. The distributional nature of $k(\alpha, \beta)$ is spelled out in the following.

Lemma 3.1. Let $u$ be the solution (unique up to a constant) to a solvable Poisson problem $\Delta u=g$. (Here $g$ might be $g_{\phi}$ or $g_{\phi}-\widehat{g}_{\phi}$ for a nonzero average $\widehat{g}_{\phi}$, but in any case we drop the subscript $\phi$ for the lemma.) We then have

$$
\int_{S^{2}} \mathrm{~d} \Omega k(\alpha, \beta) g(\alpha, \beta)=4 \int_{0}^{2 \pi} \mathrm{d} \beta \cos \beta u^{\prime}(0, \beta),
$$

where the prime in (3.14) stands for $\partial / \partial \alpha$ differentiation. Before turning to the proof, we note that Appendix [C further justifies (3.14) with a formal argument based on the machinery of scalar spherical harmonics.

Proof. We define the angular region $S_{\varepsilon}^{2}=\{(\alpha, \beta): 0 \leq \beta<2 \pi, 0 \leq \alpha \leq \varepsilon\}$ about the North Pole. Consider two such regions, $S_{\varepsilon}^{2}$ and $S_{\eta}^{2}$, where $\eta>\varepsilon$. Then the closure of $S_{\eta}^{2} \backslash S_{\varepsilon}^{2}$ is the spherical band $\{(\alpha, \beta): 0 \leq \beta<2 \pi, \varepsilon \leq \alpha \leq \eta\}$. Next, we integrate the identity $k(\alpha, \beta)[\Delta u(\alpha, \beta)-g(\alpha, \beta)]=0$ over $S_{\eta}^{2} \backslash S_{\varepsilon}^{2}$, shifting the Laplacian off of $u(\alpha, \beta)$ and onto $k(\alpha, \beta)$, thereby obtaining

$$
\begin{aligned}
\int_{0}^{2 \pi} \mathrm{d} \beta \sin \eta\left[k(\eta, \beta) u^{\prime}(\eta, \beta)-u(\eta, \beta) k^{\prime}(\eta, \beta)\right] \\
\quad=\int_{0}^{2 \pi} \mathrm{d} \beta \sin \varepsilon\left[k(\varepsilon, \beta) u^{\prime}(\varepsilon, \beta)-u(\varepsilon, \beta) k^{\prime}(\varepsilon, \beta)\right]+\int_{S_{\eta}^{2} \backslash S_{\varepsilon}^{2}} \mathrm{~d} \Omega k(\alpha, \beta) g(\alpha, \beta) .
\end{aligned}
$$

Now, in detail we have

$$
\begin{aligned}
\int_{0}^{2 \pi} \mathrm{d} \beta \sin \varepsilon\left[k(\varepsilon, \beta) u^{\prime}(\varepsilon, \beta)-u(\varepsilon, \beta) k^{\prime}(\varepsilon, \beta)\right] \\
\quad=\int_{0}^{2 \pi} \mathrm{d} \beta \cos \beta\left[\frac{\sin ^{2} \varepsilon}{\cos \varepsilon-1} u^{\prime}(\varepsilon, \beta)+\frac{\sin \varepsilon}{\cos \varepsilon-1} u(\varepsilon, \beta)\right] \sim-4 \int_{0}^{2 \pi} \mathrm{d} \beta \cos \beta u^{\prime}(0, \beta),
\end{aligned}
$$


with the asymptotic result holding as $\varepsilon \rightarrow 0^{+}$. In reaching the last result, we have exploited the expansion

$$
u(\varepsilon, \beta)=C+u^{\prime}(0, \beta) \varepsilon+O\left(\varepsilon^{2}\right),
$$

where, due to the regularity of $u(\alpha, \beta)$ in a neighborhood of the $\alpha=0$ North Pole, the constant $C$ has no $\beta$-dependence. Therefore, under the $\varepsilon \rightarrow 0^{+}$limit, (3.15) becomes

$$
\begin{aligned}
\int_{0}^{2 \pi} \mathrm{d} \beta \sin \eta\left[k(\eta, \beta) u^{\prime}(\eta, \beta)-u(\eta, \beta) k^{\prime}(\eta, \beta)\right] \\
\quad=-4 \int_{0}^{2 \pi} \mathrm{d} \beta \cos \beta u^{\prime}(0, \beta)+\int_{S_{\eta}^{2}} \mathrm{~d} \Omega k(\alpha, \beta) g(\alpha, \beta) .
\end{aligned}
$$

Straightaway, the $\eta \rightarrow \pi^{-}$limit of this equation proves the lemma (3.14).

At least in spirit, the lemma shows that the history term (3.4) "lives" on the ring $\Re\left(t_{1}, T-t_{1}\right)$. Indeed, since the average $\widehat{g}_{\phi}$ in (3.11) does not depend on $(\alpha, \beta)$,

$$
\int_{S^{2}} d \Omega k(\alpha, \beta) g_{\phi}(\alpha, \beta)=\int_{S^{2}} d \Omega k(\alpha, \beta)\left[g_{\phi}(\alpha, \beta)-\widehat{g}_{\phi}\right] .
$$

Therefore, since the Poisson problem $\Delta u_{\phi}=g_{\phi}-\widehat{g}_{\phi}$ is solvable, we may write

$$
\mathcal{H}\left(t_{0}, t_{1}\right)=4 \int_{0}^{2 \pi} \mathrm{d} \phi \int_{0}^{2 \pi} \mathrm{d} \beta \cos \beta u_{\phi}^{\prime}(0, \beta) .
$$

Now assuming CID initial data, we are tempted to view (3.1), with this version of the history term $\mathcal{H}\left(t_{0}, t_{1}\right)$, as determining the value $U(T, \mathbf{X})$ solely in terms of $t_{1}$-data, that is, simultaneously on the past null cone of $(T, \mathbf{X})$ and essentially within the computational domain. Such a formula would be the concrete realization of the Warchall theorem that we seek. However, we have not shown that the vanishing of $g_{\phi}(\alpha, \beta)$ in a neighborhood of the North Pole implies that $\int_{S^{2}} d \Omega k(\alpha, \beta) g_{\phi}(\alpha, \beta)$ vanishes. Appendix C further explores this central issue. Moreover, the point-by-point ring contribution stems from a family of Poisson problems, with each appearing to depend nonlocally on field values, through $g_{\phi}(\alpha, \beta)-\widehat{g}_{\phi} \stackrel{\text { CID }}{=} g_{\phi}(\alpha, \beta)$, over an entire sphere. By first computing the $\phi$-average of $g_{\phi}(\alpha, \beta)$, one can instead solve a single Poisson problem.

Note that $g_{\phi}(\alpha, \beta)$ has a special form (3.7) and stems from a solution to the wave equation. Several factors then suggest that the history term (3.4) may be localized about the ring $\Re\left(t_{1}, T-t_{1}\right)$, provided the initial data obeys our CID condition. First, the sphere $B_{\mathbf{X}\left(T-t_{0}, \phi\right)}\left(t_{1}, t_{1}-t_{0}\right)$ of integration is causally disconnected from the update point $(T, \mathbf{X})$, save for where it osculates the ring. We remark further on this issue below. Second, a certain class of point-source solutions does give rise to explicit localizations. Even for a $g_{\phi}(\alpha, \beta)$ stemming from a monopole source (and even with the monopole location chosen for simplification), establishing this localization directly from (3.4) is involved. Indeed, in following this path, we have found it necessary to split the expression for $g_{\phi}(\alpha, \beta)$ into a piece which integrates to the ring along with separate remainder terms. These remainders ultimately give rise to a (correct) term at $\Re\left(t_{0}, T-t_{0}\right)$, and so in total they vanish, provided the initial data obey our CID condition. However, as they are all individually singular on the sphere, their combination is a delicate calculation. For this reason, in Section 5 we establish the localization for point sources using another method. 
3.3. Further comments on the main formula. The domain of integration in (3.4) is determined by the spheres $B_{\mathbf{X}\left(T-t_{0}, \phi\right)}\left(t_{1}, t_{1}-t_{0}\right)$, which, for varying $\phi$, sweep out a solid spatial torus $\mathcal{T}_{\mathbf{X}}\left(t_{1}, T-t_{0}, t_{1}-t_{0}\right)$ parameterized by

$$
x=X+\left(T-t_{0}-\varrho \cos \omega\right) \cos \gamma, \quad y=Y+\left(T-t_{0}-\varrho \cos \omega\right) \sin \gamma, \quad z=Z+\varrho \sin \omega,
$$

with $0 \leq \gamma, \omega<2 \pi$ and $0 \leq \varrho \leq\left(t_{1}-t_{0}\right)$. We note that the integration in (3.4) sweeps over points in $\mathcal{T}_{\mathbf{X}}\left(t_{1}, T-t_{0}, t_{1}-t_{0}\right)$ more than once, and

$$
\Re_{\mathbf{X}}\left(t_{1}, T-t_{1}\right)=\operatorname{cl} B_{\mathbf{X}}^{+}\left(t_{1}, T-t_{1}\right) \cap \mathcal{T}_{\mathbf{X}}\left(t_{1}, T-t_{0}, t_{1}-t_{0}\right) .
$$

The set $\mathcal{T}_{\mathbf{X}}\left(t_{1}, T-t_{0}, t_{1}-t_{0}\right) \backslash \Re_{\mathbf{X}}\left(t_{1}, T-t_{1}\right)$ has no casual influence on the update point $(T, \mathbf{X})$, which is remarkable in light of the identity

$$
\left(T-t_{1}\right)\left\langle U_{t}\right\rangle_{B_{\mathbf{X}}^{+}\left(t_{1}, T-t_{1}\right)}+\partial_{T}\left(T-t_{1}\right)\langle U\rangle_{B_{\mathbf{X}}^{+}\left(t_{1}, T-t_{1}\right)} \stackrel{\text { CID }}{=} \mathcal{H}\left(t_{0}, t_{1}\right),
$$

with (3.4) on the right-hand side. This formula arises from comparison of (3.1) with the ordinary spherical means formula. For initial data obeying the CID condition it relates integration over two sets which are causally disconnected, apart from the intersection $\Re_{\mathbf{X}}\left(t_{1}, T-t_{1}\right)$ of their closures (a set of measure zero with respect to both).

Given data $\left\{U\left(t_{0}, \mathbf{x}\right), U_{t}\left(t_{0}, \mathbf{x}\right)\right\}$ which is supported only for $z<Z$, consider a perturbation $\left\{\delta U\left(t_{0}, \mathbf{x}\right), \delta U_{t}\left(t_{0}, \mathbf{x}\right)\right\}$ of the data. We may confine the perturbation's support to a region $\mathcal{E}$ of space that lies outside of the backward null cone of $(T, \mathbf{X})$, but does influence $U\left(t_{1}, \mathbf{x}\right)$ and $U_{t}\left(t_{1}, \mathbf{x}\right)$ on $\mathcal{T}_{\mathbf{X}}\left(t_{1}, T-t_{0}, t_{1}-t_{0}\right) \backslash \Re_{\mathbf{X}}\left(t_{1}, T-t_{1}\right)$. Moreover, taking $\mathcal{E}^{-}$as the half of $\mathcal{E}$ for which $z<Z$, we may further restrict the perturbation's support to the interior $\operatorname{int}\left(\mathcal{E}^{-}\right)$of $\mathcal{E}^{-}$. Although we shall not describe the relevant spatial region $\operatorname{int}\left(\mathcal{E}^{-}\right)$, we do show a cross section of $\mathcal{E}$ in Figure 2, The evolution of a perturbation supported on $\operatorname{int}\left(\mathcal{E}^{-}\right)$, when substituted into (3.4), must yield zero. Clearly, such appropriately supported data yields zero when substituted into the original history term (3.2).

Let us turn to another observation. Before in (3.7) we suppressed the dependence of $g_{\phi}(\alpha, \beta)$ on $t_{1}, T-t_{0}$, and $t_{1}-t_{0}$, but now we make this dependence manifest with the explicit notation

$$
g_{\phi}(\alpha, \beta, t, \xi, R)=-\frac{R}{4 \pi} U_{t}(t, \mathbf{X}(\xi, \phi)+R \boldsymbol{\mu}(\phi, \alpha, \beta))+\frac{\partial}{\partial R} \frac{R}{4 \pi} U(t, \mathbf{X}(\xi, \phi)+R \boldsymbol{\mu}(\phi, \alpha, \beta)) .
$$

Upon performing the $R$ differentiation and evaluating the resulting expression on $t=t_{1}$, $\xi=T-t_{0}$, and $R=\left(t_{1}-t_{0}\right)$, we see that $g_{\phi}\left(\alpha, \beta, t_{1}, T-t_{0}, t_{1}-t_{0}\right)$ is precisely (3.7). We then have the following.

Lemma 3.2. The $t_{1}$ time derivative of (3.7) is

$$
\partial_{t_{1}} g_{\phi}\left(\alpha, \beta, t_{1}, T-t_{0}, t_{1}-t_{0}\right)=-\frac{1}{4 \pi\left(t_{1}-t_{0}\right)} \Delta U\left(t_{1}, \mathbf{X}\left(T-t_{0}, \phi\right)+\left(t_{1}-t_{0}\right) \boldsymbol{\mu}(\phi, \alpha, \beta)\right),
$$

where $\Delta$ is the spherical Laplacian (3.12).

Proof. We proceed symbolically, noting that $g_{\phi}(\alpha, \beta, t, \xi, R)$ has the form

$$
\mathrm{g}_{\phi}=\frac{R}{4 \pi}\left(-\mathrm{U}_{t}+\mathrm{U}_{R}+\mathrm{U} / R\right)
$$




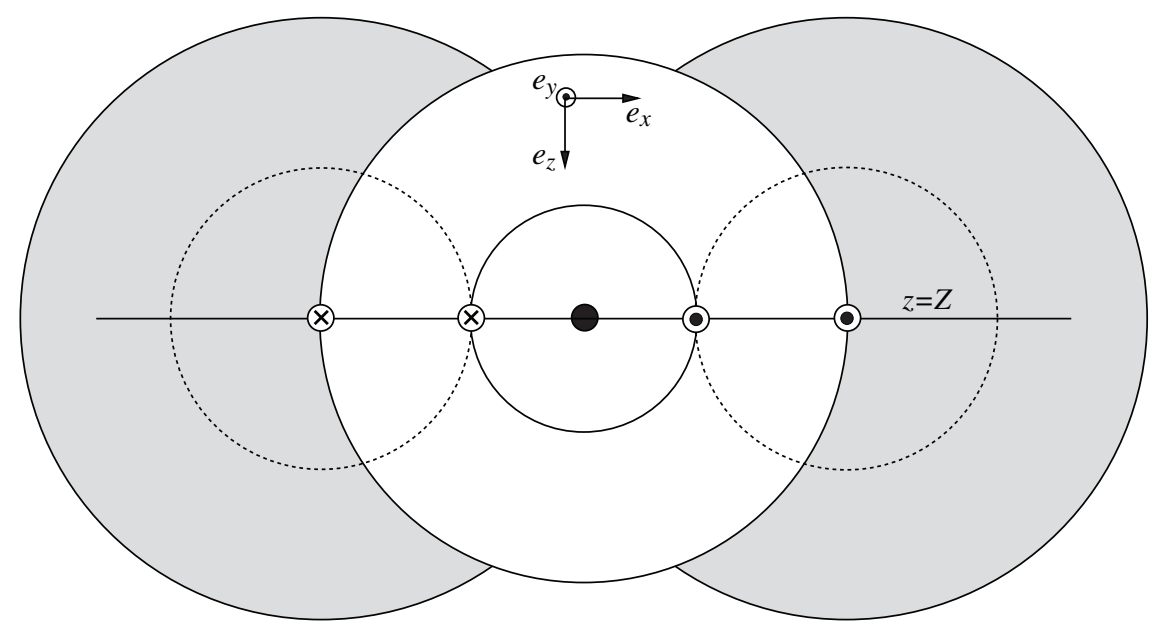

Fig. 2. Shaded Regions of influence. The figure depicts the $y=$ $Y$ plane. The heavy black dot is $\mathbf{X}=(X, Y, Z)$, and the large circle centered around $\mathbf{X}$ is a cross section of the sphere $r=T-t_{0}$. The inner $\odot$ and $\otimes$ represent the ring $\Re_{\mathbf{X}}\left(t_{1}, T-t_{1}\right)$ coming out of and going into the page, and the outer $\odot$ and $\otimes$ the ring $\Re_{\mathbf{X}}\left(t_{0}, T-t_{0}\right)$. The shaded regions are cross sections of a spatial region $\mathcal{E}$. Data supported on the interior of the set $\mathcal{E}$ influences $\mathcal{T}_{\mathbf{X}}\left(t_{1}, T-t_{0}, t_{1}-t_{0}\right)$, but has no influence on the point $(T, \mathbf{X})$. The set $\operatorname{int}\left(\mathcal{E}^{-}\right)$is that portion of $\operatorname{int}(\mathcal{E})$ for which $z<Z$.

where the expression sits at time level $t_{1}$. In (3.25) we understand that the expression (3.24) is first evaluated at $R=t_{1}-t_{0}$ and subsequently differentiated with respect to $t_{1}$. So the differentiation "sees" the $R$ slot. Therefore, the action of $\partial_{t_{1}}$ in (3.7) corresponds, in our symbolic expression (3.26), to the action of $\partial_{t}+\partial_{R}$. Since

$$
\left(\partial_{t}+\partial_{R}\right) \mathrm{g}_{\phi}=\frac{R}{4 \pi}\left(-\mathrm{U}_{t t}+\mathrm{U}_{R R}+2 \mathrm{U}_{R} / R\right)
$$

we may invoke the wave equation itself, written in spherical polar coordinates, to get the lemma. This is essentially the same argument that went into the proof of the sphericalpolygon lemma in Section 2 .

A combination of Lemmas 3.1 and 3.2 shows that

$$
\begin{aligned}
& \partial_{t_{1}} \mathcal{H}\left(t_{0}, t_{1}, T-t_{0}, t_{1}-t_{0}\right)= \\
& -\left.\frac{1}{\pi\left(t_{1}-t_{0}\right)} \int_{0}^{2 \pi} \mathrm{d} \phi \frac{\partial}{\partial \alpha} \int_{0}^{2 \pi} \mathrm{d} \beta \cos \beta U\left(t_{1}, \mathbf{X}\left(T-t_{0}, \phi\right)+\left(t_{1}-t_{0}\right) \boldsymbol{\mu}(\phi, \alpha, \beta)\right)\right|_{\alpha=0},
\end{aligned}
$$

where now the dependence of the history term $\mathcal{H}\left(t_{0}, t, \xi, R\right)$ on the extra variables is manifest. The relevant operations in (3.28) are taken in the following order: $\beta$-averaging, $\alpha$-differentiation, setting of $\alpha=0$. This formula explicitly demonstrates that if $U\left(t_{1}, \mathbf{x}\right)$ vanishes in a spatial neighborhood of the ring $\Re\left(t_{1}, T-t_{1}\right)$, then the $t_{1}$-derivative of the history term vanishes. This is necessary for consistency with the spherical-polygon lemma in Section 2. In the proof of that lemma, we have calculated, provided that 
$U\left(t_{1}, \mathbf{x}\right)$ vanishes in a neighborhood of $\Re\left(t_{1}, T-t_{1}\right)$, the value zero for the $t_{1}$-derivative

$$
\partial_{t_{1}}\left(T-t_{1}\right)\left\langle U_{t}\right\rangle_{B_{\mathbf{X}}^{-}\left(t_{1}, T-t_{1}\right)}+\partial_{t_{1}} \partial_{T}\left(T-t_{1}\right)\langle U\rangle_{B_{\mathbf{X}}^{-}\left(t_{1}, T-t_{1}\right)}
$$

of the hemispherical means contribution to $U(T, \mathbf{X})$. Since the sum of both derivatives must be zero (for $U(T, \mathbf{X})$ does not depend on $t_{1}$ ) if either derivative vanishes, then so must the other.

4. Derivation of Eq. (3.4). To derive (3.4) from (3.2), we use the future spherical means formula, which we first quickly review.

4.1. Single time level formula. To begin, we reconsider the past spherical means formula (2.11) in different symbols,

$$
U(s, \mathbf{x})=\frac{(s-t)}{4 \pi} \int_{S^{2}} \mathrm{~d} \Omega U_{t}(t, \mathbf{x}+(s-t) \boldsymbol{\nu})+\frac{\partial}{\partial s} \frac{(s-t)}{4 \pi} \int_{S^{2}} \mathrm{~d} \Omega U(t, \mathbf{x}+(s-t) \boldsymbol{\nu}),
$$

here with $s>t$. This formula expresses the field value $U(s, \mathbf{x})$ in terms of data in the past. The corresponding future spherical means formula,

$$
U(t, \mathbf{x})=-\frac{(s-t)}{4 \pi} \int_{S^{2}} \mathrm{~d} \Omega U_{t}(s, \mathbf{x}+(s-t) \boldsymbol{\nu})-\frac{\partial}{\partial t} \frac{(s-t)}{4 \pi} \int_{S^{2}} \mathrm{~d} \Omega U(s, \mathbf{x}+(s-t) \boldsymbol{\nu}),
$$

expresses the field value $U(t, \mathbf{x})$ in terms of data in the future. For our work, a more useful form of (4.2) is

$$
U(t, \mathbf{x})=-\frac{(s-t)}{2 \pi} \int_{S^{2}} \mathrm{~d} \Omega U_{t}(s, \mathbf{x}+(s-t) \boldsymbol{\nu})+\frac{\partial}{\partial s} \frac{(s-t)}{4 \pi} \int_{S^{2}} \mathrm{~d} \Omega U(s, \mathbf{x}+(s-t) \boldsymbol{\nu}) .
$$

Since the Cartesian derivative $U_{z}$ also obeys the wave equation, using (4.3), we can rewrite the integrand of $\mathcal{H}\left(t_{0}, t_{1}\right)$ in (3.2) as

$$
\begin{array}{r}
-U_{z}(\tau, \mathbf{X}(T-\tau, \phi))=\frac{\left(t_{1}-\tau\right)}{2 \pi} \int_{S^{2}} \mathrm{~d} \Omega U_{t z}\left(t_{1}, \mathbf{X}(T-\tau, \phi)+\left(t_{1}-\tau\right) \boldsymbol{\nu}\right) \\
-\frac{\partial}{\partial t_{1}}\left[\frac{\left(t_{1}-\tau\right)}{4 \pi} \int_{S^{2}} \mathrm{~d} \Omega U_{z}\left(t_{1}, \mathbf{X}(T-\tau, \phi)+\left(t_{1}-\tau\right) \boldsymbol{\nu}\right)\right]
\end{array}
$$

where $\mathbf{X}(\rho, \phi)=(X+\rho \cos \phi, Y+\rho \sin \phi, Z)$ with the notation from (3.3). Substitution of (4.4) into $\mathcal{H}\left(t_{0}, t_{1}\right)$ from (3.2) yields the following:

$$
\begin{array}{r}
\mathcal{H}\left(t_{0}, t_{1}\right)=\int_{t_{0}}^{t_{1}} \mathrm{~d} \tau \int_{0}^{2 \pi} \mathrm{d} \phi\left\{\frac{\left(t_{1}-\tau\right)}{2 \pi} \int_{S^{2}} \mathrm{~d} \Omega U_{t z}\left(t_{1}, \mathbf{X}(T-\tau, \phi)+\left(t_{1}-\tau\right) \boldsymbol{\nu}\right)\right. \\
\left.-\frac{\partial}{\partial t_{1}}\left[\frac{\left(t_{1}-\tau\right)}{4 \pi} \int_{S^{2}} \mathrm{~d} \Omega U_{z}\left(t_{1}, \mathbf{X}(T-\tau, \phi)+\left(t_{1}-\tau\right) \boldsymbol{\nu}\right)\right]\right\},
\end{array}
$$

an expression which depends solely on data at time level $t_{1}$.

4.2. Explicit $\tau$-integration. Our goal is to explicitly carry out the $\tau$-integration in (4.5). We focus first on the generic expression

$$
\mathcal{I}(\phi)=\int_{t_{0}}^{t_{1}-\varepsilon} \mathrm{d} \tau\left(t_{1}-\tau\right) \int_{S^{2}} \mathrm{~d} \Omega W_{z}\left(t_{1}, \mathbf{X}(T-\tau, \phi)+\left(t_{1}-\tau\right) \boldsymbol{\nu}\right),
$$




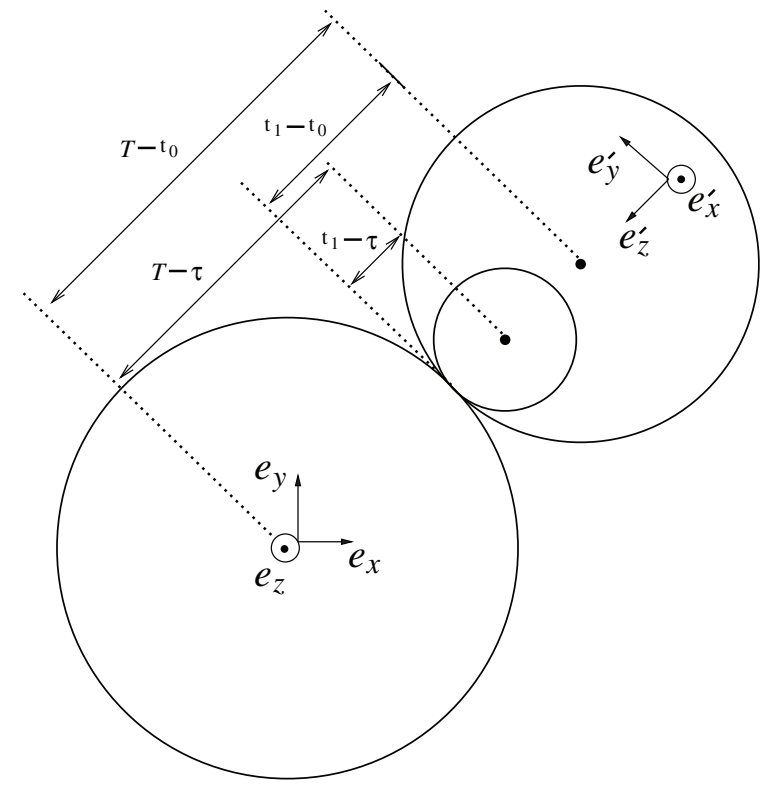

Fig. 3. Rotation of the Spherical integration. We have placed the new $z$-axis through the centers of the spheres shown. The outward-pointing unit vector for a level- $\tau$ sphere is $\boldsymbol{\nu}=\nu_{x} e_{x}+$ $\nu_{y} e_{y}+\nu_{z} e_{z}=\nu_{x}^{\prime} e_{x}^{\prime}+\nu_{y}^{\prime} e_{y}^{\prime}+\nu_{z}^{\prime} e_{z}^{\prime}$.

where $W_{z}$ is either $-U_{z} /(4 \pi)$ or $U_{t z} /(2 \pi)$ and $\varepsilon$ is a small parameter. As the surface element $d \Omega$ is rotationally invariant, we may choose the direction cosines to be

$$
\begin{aligned}
\mu_{x} & \equiv\left(\mathrm{R}_{\phi} \boldsymbol{\nu}^{\prime}\right)_{x}=-\cos \phi \nu_{z}^{\prime}-\sin \phi \nu_{y}^{\prime}=-\cos \phi \cos \alpha-\sin \phi \sin \alpha \sin \beta, \\
\mu_{y} & \equiv\left(\mathrm{R}_{\phi} \boldsymbol{\nu}^{\prime}\right)_{y}=-\sin \phi \nu_{z}^{\prime}+\cos \phi \nu_{y}^{\prime}=-\sin \phi \cos \alpha+\cos \phi \sin \alpha \sin \beta, \\
\mu_{z} & \equiv\left(\mathrm{R}_{\phi} \nu^{\prime}\right)_{z}=\nu_{x}^{\prime}=\sin \alpha \cos \beta,
\end{aligned}
$$

here in terms of new direction $\operatorname{cosines} \nu^{\prime}=(\sin \alpha \cos \beta, \sin \alpha \sin \beta, \cos \alpha)$. In this notation $\boldsymbol{\mu}$ is shorthand for $\boldsymbol{\nu}\left(\phi, \boldsymbol{\nu}^{\prime}\right)=\mathrm{R}_{\phi} \boldsymbol{\nu}^{\prime}$. The rotation $\mathrm{R}_{\phi}$ ensures that the new $z$-axis is the line connecting $(X, Y, Z)$ and $(X+(T-\tau) \cos \phi, Y+(T-\tau) \sin \phi, Z)$, as depicted in Figure 3. For each fixed $\phi$, the spatial Cartesian coordinates are

$$
\begin{aligned}
& x(\tau, \alpha, \beta)=X+(T-\tau) \cos \phi+\left(t_{1}-\tau\right) \mu_{x}(\phi, \alpha, \beta), \\
& y(\tau, \alpha, \beta)=Y+(T-\tau) \sin \phi+\left(t_{1}-\tau\right) \mu_{y}(\phi, \alpha, \beta), \\
& z(\tau, \alpha, \beta)=Z+\left(t_{1}-\tau\right) \mu_{z}(\phi, \alpha, \beta),
\end{aligned}
$$

that is, $\mathbf{x}(\tau, \alpha, \beta)=\mathbf{X}(T-\tau, \phi)+\left(t_{1}-\tau\right) \boldsymbol{\mu}(\phi, \alpha, \beta)$. The coordinate transformation $\mathbf{x}=\mathbf{x}(\tau, \alpha, \beta)$ is nonsingular, except at both $\alpha=0$ and $\tau=t_{1}$ (which is why we have introduced the small parameter $\varepsilon$ above). By the chain rule,

$$
\frac{\partial}{\partial z}=-\frac{(1+\cos \alpha) \cos \beta}{\sin \alpha} \frac{\partial}{\partial \tau}-\frac{\cos \beta}{t_{1}-\tau} \frac{\partial}{\partial \alpha}-\frac{\sin \beta}{\left(t_{1}-\tau\right) \sin \alpha} \frac{\partial}{\partial \beta} .
$$


Next, using the remarkable identity

$$
\begin{aligned}
& \left(t_{1}-\tau\right) \sin \alpha W_{z}=-\frac{\partial}{\partial \tau}\left[\left(t_{1}-\tau\right)(1+\cos \alpha) \cos \beta W\right] \\
& \quad-\frac{\partial}{\partial \alpha}(\sin \alpha \cos \beta W)-\frac{\partial}{\partial \beta}(\sin \beta W),
\end{aligned}
$$

we arrive at the result

$$
\begin{aligned}
\mathcal{I}(\phi)= & -\left(t_{1}-\tau\right) \int_{0}^{2 \pi} \mathrm{d} \beta \int_{0}^{\pi} \mathrm{d} \alpha \\
& \times\left.(1+\cos \alpha) \cos \beta W\left(t_{1}, \mathbf{X}(T-\tau, \phi)+\left(t_{1}-\tau\right) \boldsymbol{\mu}(\phi, \alpha, \beta)\right)\right|_{t_{0}} ^{t_{1}-\varepsilon} .
\end{aligned}
$$

With this formula we see that one term from (4.5) is

$$
\begin{aligned}
\text { term } 1= & \frac{\left(t_{1}-t_{0}\right)}{2 \pi} \int_{0}^{2 \pi} \mathrm{d} \phi \int_{0}^{2 \pi} \mathrm{d} \beta \int_{0}^{\pi} \mathrm{d} \alpha \\
& \times(1+\cos \alpha) \cos \beta U_{t}\left(t_{1}, \mathbf{X}\left(T-t_{0}, \phi\right)+\left(t_{1}-t_{0}\right) \boldsymbol{\mu}(\phi, \alpha, \beta)\right) .
\end{aligned}
$$

The other contribution to (4.5) is more delicate. First, notice that formally, we may pull the $\partial / \partial t_{1}$ differentiation outside of the $\tau$-integral in the second term of (4.5), because application of the derivative on the limit of integration gives zero. Therefore, for this second term the contribution we get from the lower limit of integration is

$$
\begin{aligned}
\text { term } 2 \mathrm{a}= & -\frac{\partial}{\partial t_{1}} \frac{\left(t_{1}-t_{0}\right)}{4 \pi} \int_{0}^{2 \pi} \mathrm{d} \phi \int_{0}^{2 \pi} \mathrm{d} \beta \int_{0}^{\pi} \mathrm{d} \alpha \\
& \times(1+\cos \alpha) \cos \beta U\left(t_{1}, \mathbf{X}\left(T-t_{0}, \phi\right)+\left(t_{1}-t_{0}\right) \boldsymbol{\mu}(\phi, \alpha, \beta)\right) .
\end{aligned}
$$

We remark that our main purpose in performing the rotation (4.7) has been to achieve a careful analysis of the terms which arise from the upper limit of integration $t_{1}$. The foliation of $\mathbb{R}^{3}$ into level- $\tau$ surfaces (spheres) is degenerate at $\tau=t_{1}$, and so the upper limit of integration should be handled with care 5 The only term which potentially survives from the upper limit involves the $\partial / \partial t_{1}$ differentiation. Exploiting the small parameter $\varepsilon$ introduced before, we are able to isolate this contribution as

$$
\begin{aligned}
\operatorname{term} 2 \mathrm{~b}= & \lim _{\varepsilon \rightarrow 0^{+}} \frac{1}{4 \pi} \int_{0}^{2 \pi} \mathrm{d} \phi \int_{0}^{2 \pi} \mathrm{d} \beta \int_{0}^{\pi} \mathrm{d} \alpha \\
& \times(1+\cos \alpha) \cos \beta U\left(t_{1}, \mathbf{X}\left(T-t_{1}+\varepsilon, \phi\right)+\varepsilon \boldsymbol{\mu}(\phi, \alpha, \beta)\right),
\end{aligned}
$$

\footnotetext{
${ }^{5}$ Another option is to pass from the $(\tau, \alpha, \beta)$ system to a true orthogonal system of spherical polar coordinates within and concentric with the outermost $\tau=t_{0}$ sphere. We have also carried out this approach and found identical results.
} 
which gives a zero contribution since $U$ is sufficiently smooth. Forming (term1 + term2a + term $2 \mathrm{~b}$ ), we find that (4.5) can be expressed as

$$
\begin{aligned}
\mathcal{H}\left(t_{0}, t_{1}\right)= & \frac{\left(t_{1}-t_{0}\right)}{2 \pi} \int_{0}^{2 \pi} \mathrm{d} \phi \int_{0}^{2 \pi} \mathrm{d} \beta \int_{0}^{\pi} \mathrm{d} \alpha \\
& \times(1+\cos \alpha) \cos \beta U_{t}\left(t_{1}, \mathbf{X}\left(T-t_{0}, \phi\right)+\left(t_{1}-t_{0}\right) \boldsymbol{\mu}(\phi, \alpha, \beta)\right) \\
& -\frac{\partial}{\partial t_{1}} \frac{\left(t_{1}-t_{0}\right)}{4 \pi} \int_{0}^{2 \pi} \mathrm{d} \phi \int_{0}^{2 \pi} \mathrm{d} \beta \int_{0}^{\pi} \mathrm{d} \alpha \\
& \times(1+\cos \alpha) \cos \beta U\left(t_{1}, \mathbf{X}\left(T-t_{0}, \phi\right)+\left(t_{1}-t_{0}\right) \boldsymbol{\mu}(\phi, \alpha, \beta)\right),
\end{aligned}
$$

that is, Eq. (3.6). We now revert back to a standard set of direction cosines

$$
\boldsymbol{\nu}=(\sin \theta \cos \psi, \sin \theta \sin \psi, \cos \theta)
$$

and carry out the corresponding angular transformation to find

$$
\begin{aligned}
\mathcal{H}\left(t_{0}, t_{1}\right)= & \frac{\left(t_{1}-t_{0}\right)}{2 \pi} \int_{0}^{2 \pi} \mathrm{d} \phi \int_{0}^{2 \pi} \mathrm{d} \psi \int_{0}^{\pi} \mathrm{d} \theta \\
& \times \frac{\sin \theta \cos \theta}{1+\sin \theta \cos (\psi-\phi)} U_{t}\left(t_{1}, \mathbf{X}\left(T-t_{0}, \phi\right)+\left(t_{1}-t_{0}\right) \boldsymbol{\nu}(\theta, \psi)\right) \\
& -\frac{\partial}{\partial t_{1}} \frac{\left(t_{1}-t_{0}\right)}{4 \pi} \int_{0}^{2 \pi} \mathrm{d} \phi \int_{0}^{2 \pi} \mathrm{d} \psi \int_{0}^{\pi} \mathrm{d} \theta \\
& \times \frac{\sin \theta \cos \theta}{1+\sin \theta \cos (\psi-\phi)} U\left(t_{1}, \mathbf{X}\left(T-t_{0}, \phi\right)+\left(t_{1}-t_{0}\right) \boldsymbol{\nu}(\theta, \psi)\right) .
\end{aligned}
$$

We have numerically tested (3.1) with the history term replaced by both of the previous expressions. Without further elaboration, we note that (4.17) is not as well behaved numerically as (4.15), owing to the singularity in the angular pre-factor. Notice that points on the ring $\Re\left(t_{1}, T-t_{1}\right)$ for which $\psi-\phi= \pm \pi$ and $\theta=\pi / 2$ correspond to a singular pre-factor. Rather formally, we may now write (4.17) as (3.4). The expression for the kernel (3.5) corresponds to $-\cos \theta /(1+\sin \theta \cos (\psi-\phi))$. In the formulas 44.15, 4.17), we could let the $\partial / \partial t_{1}$ act on the first slot of $U\left(t_{1}, \bullet\right)$, and then combine that part of the second term with the first, in so doing switching the $-1 /(2 \pi)$ in the first term to $\mathrm{a}-1 /(4 \pi)$. In what remains of the second term, the $-\partial / \partial t_{1}$ differentiation can then be taken as $\partial / \partial T+\partial / \partial t_{0}$, which, as expected, annihilates $\mathcal{K}\left(T-t_{0}, \mathbf{X}-\mathbf{x}, \phi\right)$.

5. Warchall theorem for point sources. For an explicit class of solutions and the boundary types considered in this paper, this section gives a constructive proof of Warchall's result. For simplicity, we continue to work with a plane boundary, but the section's main theorem extends to edge and corner cases.

Theorem 5.1. Let $\mathbf{x}_{0}$ be a source point with $z_{0}<Z-\left(T-t_{1}\right)$, and define $\mathbf{R}=\mathbf{x}-\mathbf{x}_{0}$, $\mathbf{R}=R(\sin \theta \cos \psi, \sin \theta \sin \psi, \cos \theta)$. Note that $R=\left|\mathbf{x}-\mathbf{x}_{0}\right|$ in this section is not the $R$ of Section 3. Suppose that the finite combination

$$
U^{\text {finite }}\left(t, \mathbf{R}+\mathbf{x}_{0}\right)=\sum_{\ell=0}^{\ell_{\text {max }}} \sum_{m=-\ell}^{\ell} \psi_{\ell m}(t, R) Y_{\ell m}(\theta, \psi)
$$


of multipoles is a classical solution to the wave equation which obeys our CID condition relative to the plane boundary $z=Z$. If $U^{\text {finite }}\left(t_{1}, \mathbf{x}\right)$ and $U_{t}^{\text {finite }}\left(t_{1}, \mathbf{x}\right)$ vanish on a spatial neighborhood of the hemisphere $\operatorname{cl} B_{\mathbf{X}}^{-}\left(t_{1}, T-t_{1}\right)$, then $U^{\text {finite }}(T, \mathbf{X})=0$.

Proof. First, by John's Global Holmgren Theorem as applied the wave equation [22, vanishment of the data on a spatial neighborhood of $\operatorname{cl} B_{\mathbf{X}}^{-}\left(t_{1}, T-t_{1}\right)$ implies that $U^{\text {finite }}$ vanishes on a spacetime neighborhood of this closed hemisphere. Second, the inequality $z_{0}<Z-\left(T-t_{1}\right)$ ensures that our earlier integral identities are valid. Third, it suffices to prove the theorem for a single multipole solution $U^{\text {mult }}\left(t, \mathbf{R}+\mathbf{x}_{0}\right)=\psi_{\ell m}(t, R) Y_{\ell m}(\theta, \psi)$. Indeed, consider the following operators:

$$
\begin{aligned}
D_{k} & =\prod_{\substack{\ell=0 \\
\ell \neq k}}^{\ell_{\max }}[\ell(\ell+1)+\Delta] / \prod_{\substack{\ell=0 \\
\ell \neq k}}^{\ell_{\max }}[\ell(\ell+1)-k(k+1)], \\
Q_{k n} & =\prod_{\substack{m=-k \\
m \neq n}}^{k}[m+\mathrm{i} \partial / \partial \psi] / \prod_{\substack{m=-k \\
m \neq n}}^{k}[m-n], \quad|n| \leq k,
\end{aligned}
$$

with $\Delta$ the spherical Laplacian. By the spectral properties of the spherical harmonics,

$$
Q_{k n} D_{k} U^{\text {finite }}\left(t, \mathbf{R}+\mathbf{x}_{0}\right)=\psi_{k n}(t, R) Y_{k n}(\theta, \psi) .
$$

Therefore, the individual terms in the series must vanish on the neighborhood in question. Several lemmas which follow in this section prove the result for $U^{\text {mult }}(t, \mathbf{x})$.

In trying to prove a more general theorem for an infinite multipole expansion, one encounters the difficulty that the operators $D_{k}$ and $Q_{k n}$ may no longer be used to isolate individual multipoles. Moreover, the usual $L_{2}$ projection is also not at one's disposal, since the spacetime neighborhood on which $U^{\text {finite }}$ vanishes does not contain a round sphere enclosing the source point $\mathbf{x}_{0}$.

5.1. Monopole sources. Consider the following singlet solution to the wave equation:

$$
U^{\text {sing }}(t, x, y, z)=f(t-\epsilon R) / R, \quad R^{2}=\left(x-x_{0}\right)^{2}+\left(y-y_{0}\right)^{2}+\left(z-z_{0}\right)^{2},
$$

here with $f(u)$, for $\epsilon=1$, an arbitrary profile of retarded time and $f(v)$, for $\epsilon=-1$, an arbitrary profile of advanced time. The singlet obeys

$U_{z}^{\operatorname{sing}}(\tau, X+(T-\tau) \cos \phi, Y+(T-\tau) \sin \phi, Z)=\frac{\partial}{\partial \tau}\left[\frac{f(\tau-\epsilon \mathcal{R})}{\mathcal{R}} \mathcal{I}\left(T-\tau, \mathbf{X}-\mathbf{x}_{0}, \phi\right)\right]$,

where $\mathcal{R}=\left|\mathbf{X}(T-\tau, \phi)-\mathbf{x}_{0}\right|$ in terms of the ring parameterization (3.3) and

$$
\mathcal{I}\left(T-\tau, \mathbf{X}-\mathbf{x}_{0}, \phi\right)=\frac{-\left(Z-z_{0}\right)}{\epsilon \mathcal{R}+\epsilon(T-\tau)+\left(X-x_{0}\right) \cos \phi+\left(Y-y_{0}\right) \sin \phi} .
$$

Similar formulas hold for $U_{x}^{\text {sing }}$ and $U_{y}^{\text {sing. }}$.

\footnotetext{
${ }^{6}$ For $\epsilon=-1$ this is the same kernel as in (3.5), although here it appears in a different guise, because it uses the fiducial source point $\mathbf{x}_{0}$ rather than $\mathbf{x}$. Hence here we have used $\mathcal{I}$ rather than $\mathcal{K}$.
} 
A direct calculation establishes (5.6). By $\partial / \partial z$ differentiation and setting $z=Z$, we get

$$
U_{z}^{\operatorname{sing}}(t, x, y, Z)=-\left(Z-z_{0}\right)\left[\frac{f(t-\epsilon R)}{R^{3}}+\frac{\epsilon f^{\prime}(t-\epsilon R)}{R^{2}}\right]
$$

here with $R$ as in (5.5). We now view

$$
U_{z}^{\text {sing }}(t, x, y, Z)=U_{z}^{\operatorname{sing}}(\tau, X+(T-\tau) \cos \phi, Y+(T-\tau) \sin \phi, Z),
$$

as in the statement of the lemma. After some work, we then express (5.8) as

$U_{z}^{\operatorname{sing}}(\tau, X+(T-\tau) \cos \phi, Y+(T-\tau) \sin \phi, Z)=-\left(Z-z_{0}\right) \frac{f(\tau-\epsilon \mathcal{R})}{\mathcal{R}^{3}}+\frac{\mathcal{I}}{\mathcal{R}} \frac{\partial}{\partial \tau} f(\tau-\epsilon \mathcal{R})$.

A combination of (5.10) with the key identity $\partial_{\tau}(\mathcal{I} / \mathcal{R})=-\left(Z-z_{0}\right) / \mathcal{R}^{3}$ yields the result (5.6). The identity (5.6) is consistent with our earlier formula (2.16). Indeed, upon substitution of (5.6) into the right-hand side of (2.16), we may carry out the $\tau$ integration. Using the result

$$
\int_{0}^{\pi} \frac{\mathrm{d} \phi}{1+a \cos \phi}=\frac{\pi}{\sqrt{1-a^{2}}}, \quad|a|<1
$$

we can then perform the leftover $\phi$-integration at $\tau=T$.

Lemma 5.2. Consider an outgoing singlet solution $U^{\operatorname{sing}}(t, \mathbf{x})$ given by (5.5) which obeys the CID condition with respect to the plane boundary $z=Z$, with the source point $\mathbf{x}_{0}$ such that $z_{0}<Z-\left(T-t_{1}\right)$. If $U^{\text {sing }}$ vanishes on a spatial neighborhood of the hemisphere $\operatorname{cl} B_{\mathbf{X}}^{-}\left(t_{1}, T-t_{1}\right)$, then $U^{\text {sing }}(T, \mathbf{X})=0$.

Proof. The claim is easily proved with (2.17). Indeed, using our $\epsilon=1$ key identity (5.6) in (2.17), we see that the history terms immediately reduce to a contribution at $t=t_{0}$ (which vanishes due to our CID condition) and one at $t=t_{1}$ (which vanishes by our assumptions).

5.2. Dipole sources. A general doublet (orbital angular momentum $\ell=1$ ) directed along $\boldsymbol{\nu}=\left(\nu_{x}, \nu_{y}, \nu_{z}\right)$ is

$$
U^{\mathrm{doub}}(t, x, y, z)=\left[\frac{f^{\prime}(t-\epsilon R)}{R}+\epsilon \frac{f(t-\epsilon R)}{R^{2}}\right] \frac{\mathbf{R} \cdot \boldsymbol{\nu}}{R},
$$

where $\mathbf{R}=\left(x-x_{0}, y-y_{0}, z-z_{0}\right)$. The dipole (5.12) arises via differentiation of the monopole solution $f(t-\epsilon R) / R$ by $\epsilon \nu^{k} \partial / \partial x_{0}^{k}$. Direct calculations establish that

$$
\begin{aligned}
U_{z}^{\text {doub }} & (\tau, X+(T-\tau) \cos \phi, Y+(T-\tau) \sin \phi, Z) \\
& =\frac{\partial}{\partial \tau}\left\{\epsilon \frac{f(\tau-\epsilon \mathcal{R})}{\mathcal{R}} \frac{\partial}{\partial \nu} \mathcal{I}\left(T-\tau, \mathbf{X}-\mathbf{x}_{0}, \phi\right)\right. \\
& \left.-\left[f^{\prime}(\tau-\epsilon \mathcal{R}) \frac{1}{\mathcal{R}} \frac{\partial \mathcal{R}}{\partial \nu}-\epsilon f(\tau-\epsilon \mathcal{R}) \frac{\partial}{\partial \nu}\left(\frac{1}{\mathcal{R}}\right)\right] \mathcal{I}\left(T-\tau, \mathbf{X}-\mathbf{x}_{0}, \phi\right)\right\} .
\end{aligned}
$$

Here $\partial / \partial \nu$ denotes the action of $\nu^{k} \partial / \partial x_{0}^{k}$. A lemma similar to Lemma 5.2 follows from (5.13), but we will now consider the general multipole case. 
5.3. General multipole sources. As shown in Appendix D a general multipole source arises via differentiation of the basic monopole source by a homogeneous differential operator corresponding to a spherical harmonic (see also [23], which established this result in the frequency domain). Therefore, the higher multipole analog of these formulas arises via suitable differentiation of (5.6) with respect to the source point $\mathbf{x}_{0}$, and with respect to a fiducial time $t_{0}$ which has to be included in the argument of $f$. After all differentiations, $t_{0}$ can be set to zero. Precisely, an order $\ell$ multipole solution is given by

$$
U^{\text {mult }}(t, x, y, z)=\left[\sum_{k=0}^{\ell} \frac{1}{R^{k+1}} \frac{\epsilon^{k}}{2^{k} k !} \frac{(\ell+k) !}{(\ell-k) !} f^{(\ell-k)}\left(t-t_{0}-\epsilon R\right)\right] Y_{\ell m}(\theta, \psi),
$$

or in the Burke formalism [24,

$$
U^{\text {mult }}(t, x, y, z)=\left\{f\left(t-t_{0}-\epsilon R\right)\right\}_{\ell} Y_{\ell m}(\theta, \psi) .
$$

Following Appendix D, we introduce operators $L_{\ell m}^{0}$ such that $t^{7}$

$$
U^{\text {mult }}(t, x, y, z)=\left\{f\left(t-t_{0}-\epsilon R\right)\right\}_{\ell} Y_{\ell m}(\theta, \psi)=\epsilon^{\ell} L_{\ell m}^{0} \frac{f\left(t-t_{0}-\epsilon R\right)}{R},
$$

whence formally we find

$$
\begin{aligned}
& U_{z}^{\text {mult }}(\tau, X+(T-\tau) \cos \phi, Y+(T-\tau) \sin \phi, Z) \\
& \quad=\epsilon^{\ell} \frac{\partial}{\partial \tau} L_{\ell m}^{0}\left[\frac{f\left(\tau-t_{0}-\epsilon \mathcal{R}\right)}{\mathcal{R}} \mathcal{I}\left(T-\tau, \mathbf{X}-\mathbf{x}_{0}, \phi\right)\right],
\end{aligned}
$$

as the sought-for identity.

Lemma 5.3. Consider an outgoing order- $\ell$ multipole solution $U^{\text {mult }}(t, \mathbf{x})$ given by (5.14) which obeys our CID condition with respect to the plane boundary $z=Z$, with the source point $\mathbf{x}_{0}$ such that $z_{0}<Z-\left(T-t_{1}\right)$. Provided that $U^{\text {mult }}$ vanishes on a spacetime neighborhood of the hemisphere $\operatorname{cl} B_{\mathbf{X}}^{-}\left(t_{1}, T-t_{1}\right)$, then $U^{\text {mult }}(T, \mathbf{X})=0$.

Proof. With (5.17), the result is again easily proved with (2.17), provided we first show that the underlying function $f$ vanishes for all retarded times associated with the spacetime neighborhood of $\Re_{\mathbf{X}}\left(t_{1}, T-t_{1}\right)$ in question. Since $U^{\text {mult }}(t, x, y, z)$ vanishes on this neighborhood, we have that $\left\{f\left(t-t_{0}-R\right)\right\}_{\ell}$ as given in (5.14) (5.15) vanishes for all retarded time $t-t_{0}-R$ values associated with the neighborhood. The explicit formula

$$
f\left(t-t_{0}-R\right)=(-1)^{\ell} \frac{2^{\ell}}{(2 \ell) !}\left[R^{2}\left(\partial_{t}+\partial_{R}\right)\right]^{\ell} R\left\{f\left(t-t_{0}-\epsilon R\right)\right\}_{\ell}
$$

then shows that $f\left(t-t_{0}-R\right)$ vanishes for the same retarded times.

5.4. Kirchhoff integrals. We may apply the formulas (5.6) and (5.13) in order to obtain identities for the integrand in Kirchhoff integrals. Such identities would seem a promising approach toward a sufficiently general realization of Warchall's conception. However, in our efforts along this path we have encountered an inverse problem associated with the relevant retarded potential integral equations. Nevertheless, the possibility would seem pertinent in light of the explicit $\tau$-integration carried out in Subsection 4.1. Indeed, in that analysis we represented the solution in terms of the future spherical means formula

\footnotetext{
${ }^{7}$ The discrepancy of $(-1)^{\ell}$ between the result here and (D.11) from Appendix D arises because here we differentiate with respect to the naught variables.
} 
(a special instance of the advanced Kirchhoff formula), and so as a continuum of doublets and singlets at time level $t_{1}$. Therefore, given our results for monopoles and dipoles in this section, it is not surprising that we could then carry out the $\tau$-integration explicitly. However, we stress that that scenario in Subsection 4.1 was more delicate, owing to the singular behavior on the ring and with sources on both sides of the boundary plane.

6. Edge and corner formulas. This section lists quarter and eighth spherical formulas analogous to the hemispherical formula (2.17). These formulas also feature historydependent integrals, and the analysis in Sections 4 and 5 would carry over to these integrals. For simplicity, we again choose $\mathbf{X}=(0,0,0)$, and pick definite choices for edge and corner locations. Euclidean motions would give other choices. In Subsection 6.1 the "computational domain" is $x>0, z<0$, while in Subsection 6.2 it is $x>0, y<0, z<0$.

6.1. Edge formulas. Another realization of Eq. (2.5) is the three-fourths formula

$$
\begin{aligned}
3 \pi U(T, 0,0,0) & =\int_{t}^{T} \mathrm{~d} \tau \int_{\pi / 2}^{3 \pi / 2} \mathrm{~d} \psi U_{x}(\tau, 0,(T-\tau) \sin \psi,(T-\tau) \cos \psi) \\
& -\int_{t}^{T} \mathrm{~d} \tau \int_{-\pi / 2}^{\pi / 2} \mathrm{~d} \psi U_{z}(\tau,(T-\tau) \cos \psi,(T-\tau) \sin \psi, 0) \\
& +(T-t) \int_{\mathcal{S}^{3 / 4}} \mathrm{~d} \Omega U_{t}(t,(T-t) \boldsymbol{\nu})+\frac{\partial}{\partial T}\left[(T-t) \int_{\mathcal{S}^{3 / 4}} \mathrm{~d} \Omega U(t,(T-t) \boldsymbol{\nu})\right]
\end{aligned}
$$

Here the integration is over the angular parameter space for three-fourths of a sphere

$$
\mathcal{S}^{3 / 4}=\mathcal{S}^{+} \cup\{(\theta, \phi): \pi / 2 \leq \theta<\pi, \pi / 2<\phi<3 \pi / 2\},
$$

where $\mathcal{S}^{+}$is defined just after (2.12). Taken together, the first two integrals on the righthand side of (6.1) constitute a path integral over a closed three-space curve which is continuous but has corners at $(x, y, z)=(0, \pm(T-t), 0)$. Eq. (6.1) makes no assumptions on the support of the initial data.

In parallel with the analysis in Subsection 2.3, we now combine (6.1) with the ordinary spherical means formula to obtain a formula analogous to (2.17). First, we assume that the $t_{0}$ initial data vanishes on a neighborhood of $\mathrm{cl}^{3 / 4}\left(t_{0}, T-t_{0}\right)$, where $B^{3 / 4}\left(t_{0}, T-\right.$ $\left.t_{0}\right)=B\left(t_{0}, T-t_{0}\right) \backslash\left\{(x, y, z): r=T-t_{0}, x \geq 0, z \leq 0\right\}$. Then (6.1) with $t=t_{0}$ yields

$$
\begin{aligned}
3 \pi U(T, 0,0,0) & \stackrel{\mathrm{CID}}{=} \int_{t_{0}}^{T} \mathrm{~d} \tau \int_{\pi / 2}^{3 \pi / 2} \mathrm{~d} \psi U_{x}(\tau, 0,(T-\tau) \sin \psi,(T-\tau) \cos \psi) \\
& -\int_{t_{0}}^{T} \mathrm{~d} \tau \int_{-\pi / 2}^{\pi / 2} \mathrm{~d} \psi U_{z}(\tau,(T-\tau) \cos \psi,(T-\tau) \sin \psi, 0) .
\end{aligned}
$$

Now an alternative interpretation of the CID equality (cf. footnote 2) would keep the appropriate integrals over $B^{3 / 4}\left(t_{0}, T-t_{0}\right)$ rather than $B^{+}\left(t_{0}, T-t_{0}\right)$. Second, we combine (6.1) with $t=t_{1}$ and the ordinary spherical means formula with data at $t=t_{1}$ to produce the "mirror formula" ("complement formula" would be more apt here) to (6.1), a formula involving angular integration over $\mathcal{S}^{1 / 4}=\operatorname{int}\left(S^{2} \backslash \mathcal{S}^{3 / 4}\right)$. Finally, we substitute this complement formula into the last equation, thereby arriving at the edge formula 
analogous to (2.17),

$$
\begin{aligned}
4 \pi U(T, 0,0,0) & \stackrel{\mathrm{CID}}{=} \int_{t_{0}}^{t_{1}} \mathrm{~d} \tau \int_{\pi / 2}^{3 \pi / 2} \mathrm{~d} \phi U_{x}(\tau, 0,(T-\tau) \sin \phi,(T-\tau) \cos \phi) \\
& -\int_{t_{0}}^{t_{1}} \mathrm{~d} \tau \int_{-\pi / 2}^{\pi / 2} \mathrm{~d} \phi U_{z}(\tau,(T-\tau) \cos \phi,(T-\tau) \sin \phi, 0) \\
& +\left(T-t_{1}\right)\left\langle U_{t}\right\rangle_{B^{1 / 4}\left(t_{1}, T-t_{1}\right)}+\partial_{T}\left(T-t_{1}\right)\langle U\rangle_{B^{1 / 4}\left(t_{1}, T-t_{1}\right)},
\end{aligned}
$$

where $B^{1 / 4}\left(t_{1}, T-t_{1}\right)=\left\{(x, y, z): r=T-t_{1}, x>0, z<0\right\}$.

6.2. Corner formulas. Yet another realization of (2.5) is the seven-eighths formula

$$
\begin{aligned}
\frac{7 \pi}{2} U(T, 0,0,0) & =\int_{t}^{T} \mathrm{~d} \tau \int_{\pi}^{3 \pi / 2} \mathrm{~d} \psi U_{x}(\tau, 0,(T-\tau) \sin \psi,(T-\tau) \cos \psi) \\
& -\int_{t}^{T} \mathrm{~d} \tau \int_{\pi / 2}^{\pi} \mathrm{d} \psi U_{y}(\tau,(T-\tau) \sin \psi, 0,(T-\tau) \cos \psi) \\
& -\int_{t}^{T} \mathrm{~d} \tau \int_{3 \pi / 2}^{2 \pi} \mathrm{d} \psi U_{z}(\tau,(T-\tau) \cos \psi,(T-\tau) \sin \psi, 0) \\
& +(T-t) \int_{\mathcal{S}^{7 / 8}} \mathrm{~d} \Omega U_{t}(t,(T-t) \boldsymbol{\nu})+\frac{\partial}{\partial T}\left[(T-t) \int_{\mathcal{S}^{7 / 8}} \mathrm{~d} \Omega U(t,(T-t) \boldsymbol{\nu})\right],
\end{aligned}
$$

where the angular parameter space is now

$$
\mathcal{S}^{7 / 8}=\mathcal{S}^{3 / 4} \cup\{(\theta, \phi): \pi / 2 \leq \theta<\pi, 0<\phi \leq \pi / 2\} .
$$

Taken as a whole, the first three integrals on the right-hand side of (6.5) constitute a path integral over a closed, continuous, three-space curve with corners (in the following order) at the coordinates $(0,-(T-t), 0),((T-t), 0,0)$, and $(0,0,-(T-t))$. Eq. (6.5) makes no assumptions on the support of the initial data.

Following a derivation similar to those already presented, one finds the following corner analog of formulas (2.17) and (6.4):

$$
\begin{aligned}
4 \pi U(T, 0,0,0) & \stackrel{\text { CID }}{=} \int_{t_{0}}^{t_{1}} \mathrm{~d} \tau \int_{\pi}^{3 \pi / 2} \mathrm{~d} \psi U_{x}(\tau, 0,(T-\tau) \sin \psi,(T-\tau) \cos \psi) \\
& -\int_{t_{0}}^{t_{1}} \mathrm{~d} \tau \int_{\pi / 2}^{\pi} \mathrm{d} \psi U_{y}(\tau,(T-\tau) \sin \psi, 0,(T-\tau) \cos \psi) \\
& -\int_{t_{0}}^{t_{1}} \mathrm{~d} \tau \int_{3 \pi / 2}^{2 \pi} \mathrm{d} \psi U_{z}(\tau,(T-\tau) \cos \psi,(T-\tau) \sin \psi, 0) \\
& +\left(T-t_{1}\right)\left\langle U_{t}\right\rangle_{B^{1 / 8}\left(t_{1}, T-t_{1}\right)}+\partial_{T}\left(T-t_{1}\right)\langle U\rangle_{B^{1 / 8}\left(t_{1}, T-t_{1}\right)} .
\end{aligned}
$$

Here $B^{1 / 8}\left(t_{1}, T-t_{1}\right)=\left\{(x, y, z): r=T-t_{1}, x>0, y<0, z<0\right\}$, and now an alternative interpretation to the CID equality (cf. footnote 2) would keep the appropriate integrals over $B^{7 / 8}\left(t_{0}, T-t_{0}\right)$.

6.3. Further comments. Starting with either (6.4) or (6.7), one may also use the future spherical means formula to produce a split time level formula for either an edge or corner which is fully analogous to (4.5). For such a more complicated formula, analogous remarks about causality and domains of integration would be apparent. The $\tau$-integration 
in all history terms can then be carried out explicitly in the same fashion as in Subsection 4.2. leading to kernels such as the one considered in Subsection 3.1.

7. Acknowledgments. I am grateful for the guidance of Professor T. Hagstrom, from whom I learned of the paper by $\mathrm{H}$. A. Warchall, and also for conversations with A. Narayan. Many of the results here arose from discussions with Professor Hagstrom.

Appendix A. Laplace-convolution plane boundary condition. This appendix shows that (2.24) is equivalent to the boundary condition of [4. We have given the same argument before in [14. For simplicity, we now take $t_{0}=0$, stop using capital letters $T, X, Y, Z$ for the evaluation point, and suspend the CID notation (cf. footnote2). Throughout this appendix, we assume that the initial data vanishes on $z \geq 0$. Moreover, we now use $\mathbf{x}$ to denote $(x, y)$, whereas before $\mathbf{x}$ denoted $(x, y, z)$.

A.1. Temporal convolution. Here set at $z=0$, the outgoing condition of [4] is

$$
U_{t}(t, x, y, 0)+U_{z}(t, x, y, 0)=\mathcal{F}^{-1}[\widehat{h}(t, \xi, \eta, 0)](x, y),
$$

where $\mathcal{F}^{-1}$ denotes Fourier inversion, $\boldsymbol{\xi}=(\xi, \eta)$ are the variables dual to $\mathbf{x}=(x, y)$, and

$$
\widehat{h}(t, \xi, \eta, 0)=\int_{0}^{t} \mathrm{~d} \tau \frac{J_{1}(w(t-\tau))}{w(t-\tau)}\left[-w^{2} \widehat{U}(\tau, \xi, \eta, 0)\right]
$$

is a temporal Laplace convolution with $w=\sqrt{\xi^{2}+\eta^{2}}$. Adopting the notation

$$
F(\xi, \eta)=\frac{1}{2 \pi} \int_{\mathbb{R}^{2}} \mathrm{~d} \mathbf{x} f(x, y) e^{-\mathrm{ix} \cdot \boldsymbol{\xi}}, \quad f(x, y)=\frac{1}{2 \pi} \int_{\mathbb{R}^{2}} \mathrm{~d} \boldsymbol{\xi} F(\xi, \eta) e^{\mathrm{i} \mathbf{x} \cdot \boldsymbol{\xi}},
$$

we write the Fourier convolution theorem in terms of $\mathbf{u}=(u, v)$ as

$$
\mathcal{F}^{-1}[F(\xi, \eta) G(\xi, \eta)](x, y)=\frac{1}{2 \pi} \int_{\mathbb{R}^{2}} \mathrm{~d} \mathbf{u} f(x-u, y-v) g(u, v) .
$$

To express (A.1) solely in physical space, we will exploit (A.4).

In order to exploit the convolution theorem, we first compute the inverse transform

$$
\frac{1}{2 \pi} \int_{\mathbb{R}^{2}} \mathrm{~d} \boldsymbol{\xi} \frac{J_{1}(w \tau)}{w \tau} e^{\mathrm{ix} \cdot \boldsymbol{\xi}}=\frac{1}{\tau} \int_{0}^{\infty} \mathrm{d} w J_{1}(w \tau) J_{0}(w \rho),
$$

where $\rho=\sqrt{x^{2}+y^{2}}$. To reach the last expression, we have switched to polar coordinates and used a well-known integral expression for $J_{0}(z)$. From Watson's monograph [25] (see Eq. (9), page 406) we then have

$$
\frac{1}{\tau} \int_{0}^{\infty} \mathrm{d} w J_{0}(w \rho) J_{1}(w \tau)=\frac{H(\tau-\rho)}{\tau^{2}},
$$

where $H(s)=0$ for $s<0, H(0)=\frac{1}{2}$, and $H(s)=1$ for $s>0$, whence we have

$$
\frac{1}{2 \pi} \int_{\mathbb{R}^{2}} \mathrm{~d} \boldsymbol{\xi} \frac{J_{1}(w \tau)}{w \tau} e^{\mathrm{ix} \cdot \boldsymbol{\xi}}=\frac{H(\tau-\rho)}{\tau^{2}}
$$

as our final expression for the desired inverse Fourier transform. 
A.2. Physical-space plane boundary condition. With the results above, we find that

$$
h(t, x, y, 0)=\frac{1}{2 \pi} \int_{0}^{t} \mathrm{~d} \tau \frac{1}{(t-\tau)^{2}} \int_{|\mathbf{x}-\mathbf{u}| \leq t-\tau} \mathrm{d} \mathbf{u} \Delta_{\mathbf{u}} U(\tau, u, v, 0) .
$$

Therefore, we can use the divergence theorem to rewrite A.1 as

$$
\begin{aligned}
& U_{t}(t, x, y, 0)+U_{z}(t, x, y, 0) \\
& \quad=\frac{1}{2 \pi} \int_{0}^{t} \mathrm{~d} \tau \frac{1}{(t-\tau)} \frac{\partial}{\partial t} \int_{0}^{2 \pi} \mathrm{d} \phi U(\tau, x+(t-\tau) \cos \phi, y+(t-\tau) \sin \phi, 0)
\end{aligned}
$$

that is, the same boundary condition as (2.24).

Appendix B. Foliation of $\mathbb{E}^{3}$ into level- $\tau$ slices. In this appendix we study the foliation of Euclidean three-space $\mathbb{E}^{3}$ (that is, $\mathbb{R}^{3}$ equipped with the Euclidean metric) by level- $\tau$ spheres, as determined by the coordinate transformation (4.8). We have a separate transformation $\mathbf{x}=\mathbf{x}(\tau, \alpha, \beta)$ for each choice of $\phi$, and each is adapted to the ring $\Re_{\mathbf{X}}\left(t_{1}, T-t_{1}\right)$ obeying $z=Z$ and $(x-X)^{2}+(y-Y)^{2}=\left(T-t_{1}\right)^{2}$ in the following way. Let $\ell_{\phi}$ be the line tangent to $\Re_{\mathbf{X}}\left(t_{1}, T-t_{1}\right)$ at the point $(x, y, z)=$ $\left(X+\left(T-t_{1}\right) \cos \phi, Y+\left(T-t_{1}\right) \sin \phi, Z\right)$, and let $H_{\phi}$ be the half-plane which lies to the side of $\ell_{\phi}$ not containing $\Re_{\mathbf{X}}\left(t_{1}, T-t_{1}\right)$. Then the coordinates $(\tau, \alpha, \beta)$ corresponding to the chosen $\phi$ cover $H_{\phi}$ (but do not cover $\mathbb{R}^{3} \backslash H_{\phi}$ ). Moreover, the collection of level- $\tau$ spheres is a smooth foliation of $H_{\phi}$, one exhibiting singular behavior in the approach toward the point $\left(X+\left(T-t_{1}\right) \cos \phi, Y+\left(T-t_{1}\right) \sin \phi, Z\right)$ of tangency. For each level- $\tau$ sphere the approach towards this point corresponds to the $\alpha \rightarrow 0^{+}$limit.

B.1. ADM form of the line-element. To study this foliation, we write the Euclidean line-element $\mathrm{d} s^{2}=\delta_{i j} \mathrm{~d} x^{i} \mathrm{~d} x^{j}$ (where $\delta_{i j}$ is the Kronecker symbol) in an Arnowit-DeserMisner form [19] with respect to the coordinates $(\tau, \alpha, \beta)$,

$$
\mathrm{d} s^{2}=M^{2} \mathrm{~d} \tau^{2}+\sigma_{a b}\left(\mathrm{~d} \chi^{a}+W^{a} \mathrm{~d} \tau\right)\left(\mathrm{d} \chi^{b}+W^{b} \mathrm{~d} \tau\right) .
$$

We are using $x^{i}$ to denote three-dimensional Cartesian coordinates $\left(x^{1}, x^{2}, x^{3}\right)=(x, y, z)$ and $\chi^{a}$ to denote the two-dimensional surface coordinates $\left(\chi^{2}, \chi^{3}\right)=(\alpha, \beta)$, reserving $\chi^{1}$ for use as $\tau$. For the case at hand, the two-metric $\sigma_{a b}$ corresponds to a round sphere of radius $\left(t_{1}-\tau\right)$, that is,

$$
\sigma_{22}=\left(t_{1}-\tau\right)^{2}, \quad \sigma_{33}=\left(t_{1}-\tau\right)^{2} \sin ^{2} \alpha, \quad \sigma_{23}=\sigma_{32}=0 .
$$

Moreover, for the "radial lapse" $M$ and "radial shift vector" $W^{a}$ we find

$$
M=(1-\cos \alpha), \quad W^{2}=-\frac{\sin \alpha}{\left(t_{1}-\tau\right)}, \quad W^{3}=0
$$

On each level- $\tau$ sphere the lapse and shift approach zero as $\alpha \rightarrow 0^{+}$, indicating a degenerate foliation. 
B.2. Orthonormal framing. It proves convenient to introduce an orthonormal coframe (a cotriad in fact), which we read off from the line-element (B.1). Boldface, lowercase, and Latin letters from the middle of the alphabet, $\mathbf{i}, \mathbf{j}, \mathbf{k}, \ldots$, will serve as the "name indices" for the cotriad, and they will range over $\mathbf{1}, \mathbf{a}=\mathbf{2}, \mathbf{3}$. We write the cotriad as 8

$$
E^{\mathbf{1}}=M \mathrm{~d} \tau, \quad E^{\mathbf{a}}=\vartheta^{\mathbf{a}}{ }_{b}\left(\mathrm{~d} \chi^{b}+W^{b} \mathrm{~d} \tau\right) .
$$

Here $\vartheta^{2}=\left(t_{1}-\tau\right) \mathrm{d} \alpha$ and $\vartheta^{\mathbf{3}}=\left(t_{1}-\tau\right) \sin \alpha \mathrm{d} \beta$ comprise the standard coframe (a codyad) for a round sphere with radius $\left(t_{1}-\tau\right)$. This codyad is dual to the standard dyad $\vartheta_{\mathbf{1}}=\left(t_{1}-\tau\right)^{-1} \partial / \partial \alpha$ and $\vartheta_{\mathbf{2}}=\left[\left(t_{1}-\tau\right) \sin \alpha\right]^{-1} \partial / \partial \beta$. As indicated, the name indices a for the dyad run over 2, 3. The triad dual to (B.4) is then

$$
E_{\mathbf{1}}=\frac{1}{M}\left(\frac{\partial}{\partial \tau}-W^{b} \frac{\partial}{\partial \chi^{b}}\right), \quad E_{\mathbf{a}}=\vartheta_{\mathbf{a}}{ }^{b} \frac{\partial}{\partial \chi^{b}} .
$$

For the case at hand, we find the explicit cotriad

$$
E^{\mathbf{1}}=(1-\cos \alpha) \mathrm{d} \tau, \quad E^{2}=\left(t_{1}-\tau\right) \mathrm{d} \alpha-\sin \alpha \mathrm{d} \tau, \quad E^{\mathbf{3}}=\left(t_{1}-\tau\right) \sin \alpha \mathrm{d} \beta,
$$

which is dual to the explicit triad

$$
E_{\mathbf{1}}=\frac{1}{1-\cos \alpha}\left[\frac{\partial}{\partial \tau}+\frac{\sin \alpha}{\left(t_{1}-\tau\right)} \frac{\partial}{\partial \alpha}\right], E_{\mathbf{2}}=\frac{1}{\left(t_{1}-\tau\right)} \frac{\partial}{\partial \alpha}, E_{\mathbf{3}}=\frac{1}{\left(t_{1}-\tau\right) \sin \alpha} \frac{\partial}{\partial \beta} .
$$

These basis vectors serve as useful tools for examining the degenerate foliation. On each level $-\tau$ sphere, the set $\left\{E_{\mathbf{2}}, E_{\mathbf{3}}\right\}$ is the standard spherical frame, and $E_{\mathbf{1}}$ is the standard inward-pointing normal. These features of the triad are preserved in the $\alpha \rightarrow 0^{+}$limit.

B.3. Connection coefficients. The affine connection on $\mathbb{E}^{3}$ is of course flat and torsion free. Nevertheless, with respect to the chosen triad (frame field), some of the associated orthonormal connection coefficients are nonzero (albeit "pure-gauge"). Using the first Cartan structure equation (no torsion)

$$
\mathrm{d} E^{\mathbf{i}}=-\omega^{\mathbf{i}} \mathbf{j} \wedge E^{\mathbf{j}},
$$

with $\omega^{\mathbf{i}} \mathbf{j}=\omega^{\mathbf{i}}{ }_{\mathbf{j} k} E^{\mathbf{k}}$ the connection one-forms, we may compute the coefficients $\omega_{\mathbf{i j k}}=$ $\omega_{[\mathbf{i j}] \mathbf{k}}$. With the antisymmetry in the first pair of indices, all nonzero coefficients stem from the following list:

$$
\omega_{\mathbf{2 1 1}}=-\frac{1}{\left(t_{1}-\tau\right)} \frac{\partial}{\partial \alpha} \log (1-\cos \alpha), \quad \omega_{\mathbf{3 2 3}}=\frac{\cot \alpha}{t_{1}-\tau}, \quad \omega_{\mathbf{2 1 2}}=\omega_{\mathbf{3 1 3}}=\frac{1}{\left(t_{1}-\tau\right)} .
$$

The last two coefficients specify the correct second fundamental form for a round sphere of radius $\left(t_{1}-\tau\right)$ embedded in $\mathbb{E}^{3}$. The first one specifies the $E_{\mathbf{2}}$-component

$$
b^{2}=-\frac{\sin \alpha}{\left(t_{1}-\tau\right)(1-\cos \alpha)}
$$

of the "acceleration" vector $b^{a} \partial / \partial \chi^{a}=b^{\mathbf{a}} \vartheta_{\mathbf{a}}$ for the $E_{\mathbf{1}}$ normal (the other component $b^{\mathbf{3}}$ is zero). Notice that the acceleration becomes infinite as $\alpha \rightarrow 0^{+}$.

\footnotetext{
8 The stem letter $E$ is commonly used for triads in the general relativity literature.
} 
B.4. Nature of the degenerate foliation. To study the degenerate nature of the foliation as $\alpha \rightarrow 0^{+}$, we first note that the norm of $\partial / \partial \tau$ is given by $\delta(\partial / \partial \tau, \partial / \partial \tau)$, that is,

$$
\delta_{\tau \tau}=M^{2}+\sigma_{a b} W^{a} W^{b}=2(1-\cos \alpha)
$$

which tends to zero as $\alpha \rightarrow 0^{+}$. In order to preserve the $\partial / \partial \tau$ direction in this limit, we define

$$
\widetilde{E}_{\mathbf{1}} \equiv \frac{1}{\sqrt{2(1-\cos \alpha)}} \frac{\partial}{\partial \tau}
$$

Then, using (B.7), we calculate that

$$
\widetilde{E}_{\mathbf{1}}=\sqrt{\frac{1-\cos \alpha}{2}} E_{\mathbf{1}}-\sqrt{\frac{1+\cos \alpha}{2}} E_{\mathbf{2}}=\cos \left(\frac{1}{2} \eta\right) E_{\mathbf{1}}+\sin \left(\frac{1}{2} \eta\right) E_{\mathbf{2}},
$$

with $\eta=2 \arctan [-(1+\cos \alpha) / \sin \alpha]$. As mentioned, for a fixed level $-\tau$ sphere, the directions $E_{\mathbf{1}}$ and $E_{\mathbf{2}}$ are well defined (inward-pointing normal and tangential direction along latitude). Therefore, we see that $\widetilde{E}_{\mathbf{1}}$ tends toward the tangential direction $-E_{\mathbf{2}}$ along a level- $\tau$ sphere as $\alpha \rightarrow 0^{+}$. Notice also that the angle $\eta$ does not depend on $\tau$, whence there is no $\tau$-dependent rotation occurring in the tangent space at the degenerate point. From (4.9) we find that

$$
\partial / \partial z=-\sin \alpha \cos \beta E_{\mathbf{1}}+\cos \alpha \sin \beta E_{\mathbf{2}}-\sin \beta E_{\mathbf{3}},
$$

which is a well-behaved transformation as expected.

Appendix C. Further study of the kernel $k(\alpha, \beta)$ and $S^{2}$ Poisson problem. As in the relevant Lemma 3.1, we drop all the $\phi$ subscripts throughout this appendix.

C.1. Fourier modes of the $S^{2}$ Poisson problem. With $m \geq 1$, we study the $m$ th moment

$$
u_{m}(\alpha)=\int_{0}^{2 \pi} \mathrm{d} \beta \cos (m \beta) u(\alpha, \beta)
$$

of a solution to $\Delta u=g$, with $\Delta$ the $S^{2}$ Laplacian (3.12), assuming that $g(\alpha, \beta)$ vanishes for $\alpha$ less than some cutoff $\alpha_{\text {cut }}$. The $m$ th moment of the equation itself is the following ODE:

$$
\frac{\mathrm{d}}{\mathrm{d} \alpha}\left(\sin \alpha \frac{\mathrm{d} u_{m}}{\mathrm{~d} \alpha}\right)-\frac{m^{2}}{\sin \alpha} u_{m}=\sin \alpha g_{m}
$$

To solve (C.2), we first note that $\cot ^{m}(\alpha / 2)$ and $\tan ^{m}(\alpha / 2)$ are linearly independent solutions to the homogeneous equation. Notice that $\tan ^{m}(\alpha / 2)$ is finite at $\alpha=0$, while $\cot ^{m}(\alpha / 2)$ is finite at $\alpha=\pi$. The Wronskian is

$$
\tan ^{m}(\alpha / 2) \frac{\partial}{\partial \alpha} \cot ^{m}(\alpha / 2)-\cot ^{m}(\alpha / 2) \frac{\partial}{\partial \alpha} \tan ^{m}(\alpha / 2)=-\frac{2 m}{\sin \alpha} .
$$

Appealing to the theory of one-dimensional Green's functions for operators of SturmLiouville type 26, we construct the following Green's function:

$$
G(\alpha, \xi)= \begin{cases}-(2 m)^{-1} \cot ^{m}(\xi / 2) \tan ^{m}(\alpha / 2), & \text { for } 0 \leq \alpha<\xi, \\ -(2 m)^{-1} \tan ^{m}(\xi / 2) \cot ^{m}(\alpha / 2), & \text { for } \xi<\alpha \leq \pi .\end{cases}
$$


Since our interest is the region were the inhomogeneity is not supported, we have

$$
u_{m}(\alpha)=(2 m)^{-1} K_{m} \tan ^{m}(\alpha / 2), \quad \text { for } \alpha<\alpha_{\text {cut }},
$$

where we have introduced the constant

$$
K_{m}=-\int_{\alpha_{\text {cut }}}^{\pi} \mathrm{d} \xi \cot ^{m}(\xi / 2) \sin (\xi) g_{m}(\xi) .
$$

Therefore,

$$
u_{m}^{\prime}(\alpha)=\frac{1}{4} K_{m} \sec ^{2}(\alpha / 2) \tan ^{m-1}(\alpha / 2) .
$$

We see that $u_{m}^{\prime}(0)=0$, unless $m=1$, in which case $u_{1}^{\prime}(1)=\frac{1}{4} K_{1}$. For $m=1$ the integral in (C.6) features $-\cot (\xi / 2)=k(\xi, \beta) / \cos \beta$, whence, turned around, the analysis here is another confirmation of (3.14). In any case, from the vanishing of $g(\alpha, \beta)$ in a neighborhood of the North Pole we cannot conclude that $u_{1}^{\prime}(0)=0$.

C.2. Spherical harmonic expansion of the kernel. Using the machinery of scalar spherical harmonics $Y_{\ell m}(\alpha, \beta)$, we present calculations which further justify our distributional interpretation of $k(\alpha, \beta)$. We compute the value on the right-hand side of (3.14) both directly and with the kernel, finding agreement between the two methods.

To fix conventions, we define the associated Legendre functions as [26]

$$
P_{\ell}^{m}(\xi)=(-1)^{m}\left(1-\xi^{2}\right)^{\frac{m}{2}} \frac{1}{2^{\ell} \ell !} \frac{\mathrm{d}^{\ell+m}}{\mathrm{~d} \xi^{\ell+m}}\left(\xi^{2}-1\right)^{\ell},
$$

with $|m| \leq \ell$. The spherical harmonics are then

$$
Y_{\ell m}(\alpha, \beta)=N_{\ell m} P_{\ell}^{m}(\cos \alpha) e^{\mathrm{i} m \beta},
$$

where the normalization constant is

$$
N_{\ell m}=\sqrt{\frac{2 \ell+1}{4 \pi} \frac{(\ell-m) !}{(\ell+m) !}} .
$$

First, we take the expansion for the source,

$$
g(\alpha, \beta)=\sum_{\ell=1}^{\infty} \sum_{m=-\ell}^{\ell} g_{\ell m} Y_{\ell m}(\alpha, \beta),
$$

and use it to write down the formal solution to the Poisson problem,

$$
u(\alpha, \beta)=-\sum_{\ell=1}^{\infty} \sum_{m=-\ell}^{\ell} \frac{g_{\ell m}}{\ell(\ell+1)} Y_{\ell m}(\alpha, \beta) .
$$

With (C.9) and (C.10), we then calculate the following moment:

$$
\begin{aligned}
4 \int_{0}^{2 \pi} \mathrm{d} \beta \cos \beta u(\alpha, \beta)=-4 \pi \sum_{\ell=1}^{\infty} \frac{1}{\ell(\ell+1)}\left[g_{\ell 1} N_{\ell 1} P_{\ell}^{1}(\cos \alpha)\right. \\
\left.+g_{\ell,-1} N_{\ell,-1} P_{\ell}^{-1}(\cos \alpha)\right] .
\end{aligned}
$$

Upon using the widely-known result ([26])

$$
P_{\ell}^{-1}(\cos \alpha)=-\frac{(\ell-1) !}{(\ell+1) !} P_{\ell}^{1}(\cos \alpha),
$$


along with (C.10), we cast (C.13) into the form

$$
4 \int_{0}^{2 \pi} \mathrm{d} \beta \cos \beta u(\alpha, \beta)=-\sqrt{4 \pi} \sum_{\ell=1}^{\infty} \sqrt{\frac{2 \ell+1}{\ell(\ell+1)}} \frac{\left(g_{\ell 1}-g_{\ell,-1}\right)}{\ell(\ell+1)} P_{\ell}^{1}(\cos \alpha) .
$$

To compute the derivative of this expression at $\alpha=0$, we first evaluate the Legendre differential equation for $P_{\ell}(\xi)$ at $\xi=1$, thereby obtaining

$$
\left.\frac{\mathrm{d}}{\mathrm{d} \alpha} P_{\ell}^{1}(\cos \alpha)\right|_{\alpha=0}=-P_{\ell}^{\prime}(1)=-\frac{\ell(\ell+1)}{2} .
$$

Finally then, with $g_{\ell 1}-g_{\ell,-1}=2 \operatorname{Re}\left(g_{\ell 1}\right)$, we arrive at

$$
4 \int_{0}^{2 \pi} \mathrm{d} \beta \cos \beta u^{\prime}(0, \beta)=\sqrt{4 \pi} \sum_{\ell=1}^{\infty} \sqrt{\frac{2 \ell+1}{\ell(\ell+1)}} \operatorname{Re}\left(g_{\ell 1}\right) .
$$

We now produce the same expression directly from the integral

$$
\int_{S^{2}} \mathrm{~d} \Omega k(\alpha, \beta) g(\alpha, \beta) .
$$

To obtain a formal expansion for the kernel, we start with the Fourier-Legendre expansion

$$
\log (1-\xi)=-1+\log 2-\sum_{\ell=1}^{\infty} \frac{2 \ell+1}{\ell(\ell+1)} P_{\ell}(\xi)
$$

which holds in the sense of mean convergence in the $L_{2}$ norm. Next, we formally differentiate (C.19), use the result $P_{\ell}^{1}(\xi)=-\sqrt{1-\xi^{2}} P_{\ell}^{\prime}(\xi)$, and appeal to (C.10), thereby finding the following formal spherical harmonic expansion for the kernel:

$$
k(\alpha, \beta)=\sqrt{4 \pi} \sum_{\ell=1}^{\infty} \sqrt{\frac{2 \ell+1}{\ell(\ell+1)}} \operatorname{Re}\left[Y_{\ell 1}(\alpha, \beta)\right] .
$$

Note the slow decay of the expansion coefficients, and further note that only $m=1$ terms appear. Eq. (C.20) does not hold in the $L_{2}$ sense, rather in the weak sense when integrated against a sufficiently smooth $g(\alpha, \beta)$, as shown by a more careful argument based on integration by parts and the expansion (C.19). The harmonic's orthonormal properties then determine that the integral (C.18) is precisely (C.17).

\section{Appendix D. Mode solutions of the $3+1$ wave equation featuring spherical} harmonics as differential operators. This appendix shows that multipole solutions of the $3+1$ wave equation arise from monopole solutions via the action of homogeneous constant-coefficient differential operators $L_{\ell m}\left(\partial_{t}, \partial_{x}, \partial_{y}, \partial_{z}\right)$ corresponding to the spherical harmonics $Y_{\ell m}(\theta, \phi)$. This appendix is essentially a time-domain version of the frequency-domain argument presented in 23 . 
D.1. Standard scalar harmonics. We begin by collecting a few standard formulas for scalar spherical harmonics. To start with, we define the associated Legendre functions as in (C.8), but now with $u$ rather than $\xi$ as the dependent variable. Via the binomial expansion, we then establish that

$$
P_{\ell}^{m}(u)=(-1)^{m}\left(1-u^{2}\right)^{\frac{m}{2}} \sum_{k=0}^{[(\ell-m) / 2]} a_{\ell m, k} u^{\ell-m-2 k}
$$

where

$$
\begin{gathered}
a_{\ell m, k}=\frac{(-1)^{k}(2 \ell-2 k) !}{2^{\ell} k !(\ell-k) !(\ell-m-2 k) !}, \\
{[(\ell-m) / 2]=\left\{\begin{array}{cc}
(\ell-m) / 2 & \text { for } \ell-m \text { even, } \\
(\ell-m-1) / 2 & \text { for } \ell-m \text { odd, }
\end{array}\right.}
\end{gathered}
$$

in agreement with Thorne's Eq. (2.8) in [27. The spherical harmonics (D.4) are then

$$
Y_{\ell m}(\theta, \phi)=(-1)^{m} N_{\ell m}\left(e^{\mathrm{i} \phi} \sin \theta\right)^{m} \sum_{k=0}^{[(\ell-m) / 2]} a_{\ell m, k}(\cos \theta)^{\ell-m-2 k},
$$

where the normalization constant appears in (C.10). Under complex conjugation the scalar harmonics behave as

$$
Y_{\ell,-m}=(-1)^{m} \bar{Y}_{\ell m} .
$$

D.2. Corresponding homogeneous operators and main lemma. For $m=|m|$ we define operators $L_{\ell m}$ associated with the $Y_{\ell m}$ by the formula

$$
L_{\ell m}=(-1)^{m} N_{\ell m}\left(\frac{\partial}{\partial x}+\mathrm{i} \frac{\partial}{\partial y}\right)^{m} \sum_{k=0}^{[(\ell-m) / 2]} a_{\ell m, k}\left(\frac{\partial}{\partial z}\right)^{\ell-m-2 k}\left(\frac{\partial}{\partial t}\right)^{2 k} .
$$

Note that this operator is constant-coefficient and degree- $\ell$ homogeneous. In parallel with (D.5) we set

$$
L_{\ell m}=(-1)^{m} \bar{L}_{\ell|m|}
$$

for $m=-|m|$.

General outgoing $(\epsilon=1)$ and ingoing $(\epsilon=-1)$ multipole solutions of the $3+1$ wave equation have the form

$$
U_{\ell m}^{\epsilon}(t, x, y, z)=\psi_{\ell}^{\epsilon}(t, r) Y_{\ell m}(\theta, \phi), \quad \psi_{\ell}^{\epsilon}(t, r)=\sum_{k=0}^{\ell} \frac{\epsilon^{k}}{r^{k+1}} \frac{1}{2^{k} k !} \frac{(\ell+k) !}{(\ell-k) !} f^{(\ell-k)}(t-\epsilon r),
$$

where $f^{(p)}(u)$ is the $p$ th derivative of an underlying function $f(u)$ of retarded time $u=$ $t-r$, and similarly for $f^{(p)}(v)$, where $v=t+r$ is advanced time. Also in (D.8) we respectively view $(x, y, z)$ as place holders for $(r \sin \theta \cos \phi, r \sin \theta \sin \phi, \cos \theta)$. Since $L_{00}=$ $Y_{00}=1 / \sqrt{4 \pi}$, we trivially find

$$
U_{00}^{\epsilon}(t, x, y, z)=L_{00} \frac{f(t-\epsilon r)}{r}=L_{00} \psi_{0}^{\epsilon}(t, r)=\psi_{0}^{\epsilon}(t, r) Y_{00}(\theta, \phi) .
$$

Our main result is the following. 
Lemma D.1. A general outgoing or ingoing multipole solution can be expressed as

$$
\psi_{\ell}^{\epsilon}(t, r) Y_{\ell m}(\theta, \phi)=(-\epsilon)^{\ell} L_{\ell m} \psi_{0}^{\epsilon}(t, r),
$$

or equivalently as

$$
U_{\ell m}^{\epsilon}(t, x, y, z)=(-\epsilon)^{\ell} L_{\ell m} r^{-1} f(t-\epsilon r) .
$$

Corollary D.2. Defining

$$
\chi_{\ell}(t, r)=\psi_{\ell}^{-1}(t, r)+(-1)^{\ell+1} \psi_{\ell}^{1}(t, r),
$$

which is regular at $r=0$, we also have the following:

$$
\chi_{\ell}(t, r) Y_{\ell m}(\theta, \phi)=L_{\ell m} \chi_{0}(t, r),
$$

a result we shall also express as

$$
U_{\ell m}^{0}(t, x, y, z)=L_{\ell m} r^{-1}[f(t+r)-f(t-r)],
$$

with $U_{\ell m}^{0}(t, x, y, z)$ representing the mode solution regular at $r=0$.

D.3. Proof of Lemma D.1, Considering the $(\ell, m)$ lattice shown in Figure 4, we follow [23. and present an inductive proof based on the following steps:

(i) Prove by induction that

$$
\psi_{\ell}^{\epsilon} Y_{\ell \ell}=(-\epsilon)^{\ell} L_{\ell \ell} \psi_{0}^{\epsilon} \quad \text { and } \quad \psi_{\ell}^{\epsilon} Y_{\ell,-\ell}=(-\epsilon)^{\ell} L_{\ell,-\ell} \psi_{0}^{\epsilon} .
$$

That is to say, establish the result for the black circles in Figure 4.

(ii) Show that the results from (i) imply

$$
\psi_{\ell}^{\epsilon} Y_{\ell+1, \ell}=(-\epsilon)^{\ell+1} L_{\ell+1, \ell} \psi_{0}^{\epsilon} \quad \text { and } \quad \psi_{\ell}^{\epsilon} Y_{\ell+1,-\ell}=(-\epsilon)^{\ell+1} L_{\ell+1,-\ell} \psi_{0}^{\epsilon} .
$$

That is to say, establish the result for the white circles in Figure 4

(iii) By induction extend the result vertically upward (at fixed $m$ ) to the crosses in Figure 4. Notice that each cross can be associated with an $(\ell+1, m)$ pair for which $(\ell, m)$ and $(\ell-1, m)$ are also lattice locations.

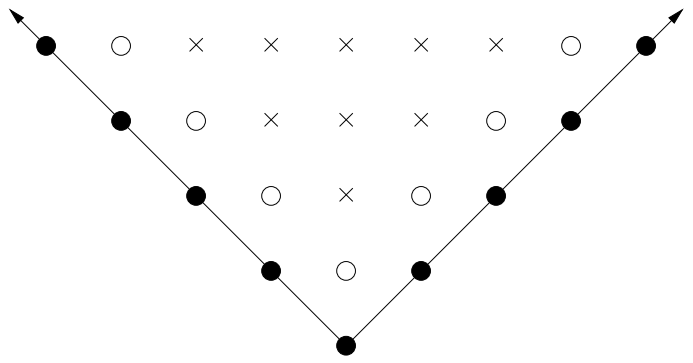

Fig. 4. Lattice $(\ell, m)$ SCAlar harmonics. In the diagram the upward vertical direction corresponds to increasing $\ell$, starting from $\ell=0$. The horizontal direction indicates each of the $2 \ell+1$ possible values of $m$. 
Before turning to the proof of each of the steps (i)-(iii), we first collect the following crucial identities:

$$
\begin{aligned}
\epsilon \psi_{\ell+1}^{\epsilon} & =\frac{\ell}{r} \psi_{\ell}^{\epsilon}-\frac{\partial \psi_{\ell}^{\epsilon}}{\partial r}, \\
\psi_{\ell+1}^{\epsilon} & =\epsilon \frac{2 \ell+1}{r} \psi_{\ell}^{\epsilon}+\frac{\partial^{2} \psi_{\ell-1}^{\epsilon}}{\partial t^{2}} .
\end{aligned}
$$

These are consequences of the explicit formula (D.8) for $\psi_{\ell}^{\epsilon}(t, r)$.

In establishing (i), it suffices to consider only $m=\ell$, since the $m=-\ell$ result follows from the $m=\ell$ result via elementary arguments based on (D.5) and (D.7). As noted in (D.9), the $\ell=m=0$ result holds trivially. We then start with (D.6), observing that

$$
L_{\ell \ell}=(-1)^{\ell} N_{\ell \ell} a_{\ell \ell, 0}\left(\frac{\partial}{\partial x}+\mathrm{i} \frac{\partial}{\partial y}\right)^{\ell},
$$

from which we infer the key recursion,

$$
L_{\ell+1, \ell+1}=-\frac{N_{\ell+1, \ell+1} a_{\ell+1, \ell+1,0}}{N_{\ell \ell} a_{\ell \ell, 0}}\left(\frac{\partial}{\partial x}+\mathrm{i} \frac{\partial}{\partial y}\right) L_{\ell \ell},
$$

upon which the induction will rest. Using (D.4), we write

$$
\psi_{\ell}^{\epsilon} Y_{\ell \ell}=(-1)^{\ell} r^{-\ell} \psi_{\ell}^{\epsilon} N_{\ell \ell} a_{\ell \ell, 0}(x+\mathrm{i} y)^{\ell} .
$$

Straightforward differentiation of this equation yields

$$
\left(\frac{\partial}{\partial x}+\mathrm{i} \frac{\partial}{\partial y}\right) \psi_{\ell}^{\epsilon} Y_{\ell \ell}=(-1)^{\ell} N_{\ell \ell} a_{\ell \ell, 0} \frac{(x+\mathrm{i} y)^{\ell+1}}{r} \frac{\partial}{\partial r}\left(\frac{\psi_{\ell}^{\epsilon}}{r^{\ell}}\right),
$$

whence by (D.15),

$$
\left(\frac{\partial}{\partial x}+\mathrm{i} \frac{\partial}{\partial y}\right) \psi_{\ell}^{\epsilon} Y_{\ell \ell}=\epsilon \frac{N_{\ell \ell} a_{\ell \ell, 0}}{N_{\ell+1, \ell+1} a_{\ell+1, \ell+1,0}} \psi_{\ell+1}^{\epsilon} Y_{\ell+1, \ell+1} .
$$

Assuming (D.10) holds at level- $\ell$ for $m=\ell$, we then combine the last equation with (D.18) to get $(-\epsilon)^{\ell+1} L_{\ell+1, \ell+1} \psi_{0}=\psi_{\ell+1} Y_{\ell+1, \ell+1}$ as expected.

Turning to step (ii), we consider only $m=\ell-1$. As before, we can get the $m=-\ell+1$ result via an argument relying on complex conjugation. We begin by noting that (D.6) implies

$$
L_{\ell+1, \ell}=-\frac{N_{\ell+1, \ell}}{N_{\ell \ell}} \frac{a_{\ell+1, \ell, 0}}{a_{\ell \ell, 0}} \frac{\partial}{\partial z} L_{\ell \ell} .
$$

Assuming the first result from (i), we then have

$$
(-\epsilon)^{\ell+1} L_{\ell+1, \ell} \psi_{0}^{\epsilon}=\epsilon \frac{N_{\ell+1, \ell}}{N_{\ell \ell}} \frac{a_{\ell+1, \ell, 0}}{a_{\ell \ell, 0}} r^{\ell} Y_{\ell \ell} \frac{\partial}{\partial z}\left(\frac{\psi_{\ell}^{\epsilon}}{r^{\ell}}\right),
$$

where in reaching this point we have used the fact that $r^{\ell} Y_{\ell \ell}$ has no $z$ dependence. An appeal to (D.15) then shows that

$$
(-\epsilon)^{\ell+1} L_{\ell+1, \ell} \psi_{0}^{\epsilon}=-\frac{N_{\ell+1, \ell}}{N_{\ell \ell}} \frac{a_{\ell+1, \ell, 0}}{a_{\ell \ell, 0}} \psi_{\ell+1}^{\epsilon} \cos \theta Y_{\ell \ell},
$$

and from this equation and (D.4) we get the desired result $(-\epsilon)^{\ell+1} L_{\ell+1, \ell} \psi_{0}^{\epsilon}=\psi_{\ell+1}^{\epsilon} Y_{\ell+1, \ell}$. 
In order to establish (iii) and finish the proof, we will make use of the following identities for the associated Legendre functions $P_{\ell}^{m}(u)$ :

$$
\begin{aligned}
(2 \ell+1) u P_{\ell}^{m} & =(\ell+m) P_{\ell-1}^{m}+(\ell-m+1) P_{\ell+1}^{m}, \\
\left(1-u^{2}\right) \frac{\mathrm{d} P_{\ell}^{m}}{\mathrm{~d} u} & =(\ell+1) u P_{\ell}^{m}-(\ell-m+1) P_{\ell+1}^{m} .
\end{aligned}
$$

One may derive these from similar identities for Legendre polynomials, which in turn stem from various differentiations of the generating function for Legendre polynomials.

From (D.15)-(D.16) and (D.25)-(D.26), we will now obtain another identity which proves crucial in establishing (iii). First, let $u=\cos \theta=z / r$, so that

$$
\frac{\partial}{\partial z}=u \frac{\partial}{\partial r}+\frac{\left(1-u^{2}\right)}{r} \frac{\partial}{\partial u}
$$

and consider $\partial_{z} \psi_{\ell}^{\epsilon}(t, r) P_{\ell}^{m}(u)$. A combination of (D.27) with (D.25)-(D.26) then yields

$$
\begin{aligned}
\frac{\partial}{\partial z} \psi_{\ell}^{\epsilon} P_{\ell}^{m} & =\left[\frac{\ell+m}{2 \ell+1} \frac{\partial \psi_{\ell}^{\epsilon}}{\partial r}+\frac{(\ell+1)(\ell+m)}{2 \ell+1} \frac{\psi_{\ell}^{\epsilon}}{r}\right] P_{\ell-1}^{m} \\
& +\left[\frac{\ell-m+1}{2 \ell+1} \frac{\partial \psi_{\ell}^{\epsilon}}{\partial r}-\frac{\ell(\ell-m+1)}{2 \ell+1} \frac{\psi_{\ell}^{\epsilon}}{r}\right] P_{\ell+1}^{m} .
\end{aligned}
$$

To obtain the desired identity, we now appeal to (D.15) $-(\underline{D .16})$, in order to rewrite the last equation as follows:

$$
\epsilon \frac{\partial}{\partial z} \psi_{\ell}^{\epsilon} P_{\ell}^{m}=-\frac{\ell+m}{2 \ell+1} \frac{\partial^{2} \psi_{\ell-1}^{\epsilon}}{\partial t^{2}} P_{\ell-1}^{m}-\frac{\ell-m+1}{2 \ell+1} \psi_{\ell+1}^{\epsilon} P_{\ell+1}^{m},
$$

from which we find

$$
\epsilon \frac{\partial}{\partial z} \psi_{\ell}^{\epsilon} Y_{\ell m}=-\frac{\ell+m}{2 \ell+1} \frac{N_{\ell m}}{N_{\ell-1, m}} \frac{\partial^{2} \psi_{\ell-1}^{\epsilon}}{\partial t^{2}} Y_{\ell-1, m}-\frac{\ell-m+1}{2 \ell+1} \frac{N_{\ell m}}{N_{\ell+1, m}} \psi_{\ell+1}^{\epsilon} Y_{\ell+1, m}
$$

Note that the desired identity (D.30) follows from (D.29), since all associated Legendre functions appearing in (D.29) have the same azimuthal index $m$.

Let us now turn to the inductive argument establishing step (iii). Rearranging (D.25) and multiplying the result by $N_{\ell+1, m} \exp (\mathrm{i} m \phi)$, we get

$$
Y_{\ell+1, m}=\frac{2 \ell+1}{\ell-m+1} \frac{N_{\ell+1, m}}{N_{\ell m}} \cos \theta Y_{\ell m}-\frac{\ell+m}{\ell-m+1} \frac{N_{\ell+1, m}}{N_{\ell-1, m}} Y_{\ell-1, m} .
$$

Notice that for the crosses in Figure 4, here labeled by $(\ell+1, m)$, we have $\ell-m+1 \neq 0$. The corresponding identity for the associated operators $L_{\ell m}$ is

$$
L_{\ell+1, m}=\frac{2 \ell+1}{\ell-m+1} \frac{N_{\ell+1, m}}{N_{\ell m}} \frac{\partial}{\partial z} L_{\ell m}-\frac{\ell+m}{\ell-m+1} \frac{N_{\ell+1, m}}{N_{\ell-1, m}} \frac{\partial^{2}}{\partial t^{2}} L_{\ell-1, m},
$$

a result which can also be obtained directly from (D.6). Applying each side of the last relationship to $(-\epsilon)^{\ell+1} \psi_{0}$, and assuming that the result (D.10) holds for levels $\ell$ and $\ell-1$, we arrive at

$$
\begin{aligned}
(-\epsilon)^{\ell+1} L_{\ell+1, m} \psi_{0}^{\epsilon}=-\epsilon \frac{2 \ell+1}{\ell-m+1} \frac{N_{\ell+1, m}}{N_{\ell m}} & \frac{\partial}{\partial z} \psi_{l}^{\epsilon} Y_{\ell m} \\
& -\frac{\ell+m}{\ell-m+1} \frac{N_{\ell+1, m}}{N_{\ell-1, m}} \frac{\partial^{2}}{\partial t^{2}} \psi_{\ell-1}^{\epsilon} Y_{\ell-1, m} .
\end{aligned}
$$


Finally then, use of (D.30) shows that

$$
(-\epsilon)^{\ell+1} L_{\ell+1, m} \psi_{0}^{\epsilon}=\psi_{\ell+1}^{\epsilon} Y_{\ell+1, m},
$$

establishing step (iii) and completing the proof.

\section{REFERENCES}

[1] T. Hagstrom and T. Warburton, A new auxiliary variable formulation of high-order local radiation boundary conditions: Corner compatibility conditions and extensions to first-order systems, Wave Motion 39 (2004) 327-338. MR2058660(2005a:35009)

[2] T. Hagstrom, T. Warburton, and D. Givoli, Radiation boundary conditions for time-dependent waves based on complete plane wave expansions, to appear in J. Comput. Appl. Math.

[3] B. Alpert, L. Greengard, and T. Hagstrom, Rapid evaluation of nonreflecting boundary kernels for time-domain wave propagation, SIAM J. Numer. Anal. 37 (2000) 1138-1164. MR1756419 (2002c:65037)

[4] B. Alpert, L. Greengard, and T. Hagstrom, Nonreflecting boundary conditions for the timedependent wave equation, J. Comput. Phys. 180 (2002) 270-296. MR.1913093 (2003e:65140)

[5] S. Jiang, Fast Evaluation of Nonreflecting Boundary Conditions for the Schrödinger Equation, New York University, Ph.D. Dissertation, 2001.

[6] S. R. Lau, Rapid evaluation of radiation boundary kernels for time-domain wave propagation on black holes: Implementation and numerical tests, Classical Quantum Gravity 21 (2004) 4147-4192. MR2100436 (2005k:83085)

[7] S. R. Lau, Rapid evaluation of radiation boundary kernels for time-domain wave propagation on blackholes: Theory and numerical methods, J. Comput. Phys. 199 (2004) 376-422. MR2081009 (2005f:83003)

[8] S. R. Lau, Analytic structure of radiation boundary kernels for blackhole perturbations, J. Math. Phys. 46 (2005) 102503, 21 pp. MR 2178591 (2006e:83099)

[9] L. Hörmander, Uniqueness theorems and wave front sets for linear differential equations with analytic coefficients, Commun. Pure Appl. Math. 24 (1971) 671-704. MR0294849 (45:3917)

[10] L. Hörmander, The Analysis of Linear Partial Differential Operators. I, Springer-Verlag, Berlin, 1990. MR.1065993 (91m:35001a)

[11] H. A. Warchall, Wave Propagation at Computational Boundaries, Commun. in Partial Differential Equations 16 (1991) 31-41. MR1096832 (92a:65251)

[12] Z.-H. Teng, Exact boundary condition for time-dependent wave equation based on boundary integral, J. Comput. Phys. 190 (2003) 398-418. MR2013024 (2004j:65127)

[13] V. H. Weston, Factorization of the wave equation in higher dimensions, J. Math. Phys. 28 (1988) 1061-1068. MR887024 (88c:35088)

[14] T. Hagstrom and S. Lau, Radiation Boundary Conditions for Maxwell's Equations: A Review of Accurate Time-domain Formulations, J. Comput. Math. 25 (2007) 305-336. MR2320236 (2008b:35289)

[15] J. Hadamard, Lectures on Cauchy's Problem in Linear Partial Differential Equations, Dover, New York, 1953. MR0051411 (14:474f)

[16] R. Courant and D. Hilbert, Methoden der mathematischen Physik II, Springer-Verlag, Berlin, 1937. MR0344038 (49:8778)

[17] B. B. Baker and E. T. Copson, The Mathematical Theory of Huygen's Principle, second edition, Oxford University Press, Oxford, 1953.

[18] M. E. Taylor, Partial Differential Equations: Basic Theory, Springer-Verlag, New York, 1996. MR.1395147 (98b:35002a)

[19] C. W. Misner, K. S. Thorne, and J. A. Wheeler, Gravitation, Freeman, New York, 1973. MR0418833 $(54: 6869)$

[20] J. Côté, M. J. Gander, L. Laayouni, and S. Loisel, Comparison of the Dirichlet-Neumann and Optimal Schwarz Method on the Sphere, Domain Decomposition Methods in Science and Engineering, Lecture Notes in Computational Science and Engineering 40, Springer-Verlag (2004) 235-242. MR2235747

[21] C. C. Lim and D. Zhi, Variational analysis of energy-enstrophy theories on the sphere, Discrete and Continuous Dynamical Systems, Supplemental Volume (2005) 611-620. MR2192720 (2006j:76063) 
[22] J. Rauch, Partial Differential Equations, first edition, Springer-Verlag, New York, 1991. MR1223093 (94e:35002)

[23] H. J. H. Clercx and P. P. J. M. Schram, An alternative expression for the addition theorems of spherical wave solutions of the Helmholtz equation, J. Math. Phys. 34 (1993) 5292-5302. MR1243141 (94m:35041)

[24] W. L. Burke, Gravitational radiation damping of slowly moving systems calculated using matched asymptotic expansions, J. Math. Phys. 12 (1971) 401-418.

[25] G. N. Watson, A Treatise on the Theory of Bessel Functions, second edition, Cambridge University Press, Cambridge, 1944. MR0010746 (6:64a)

[26] P. K. Chattopadhyay, Mathematical Physics (New Age International, Ltd., New Delhi, 1990).

[27] K. S. Thorne, Multipole moments of gravitational radiation, Rev. Mod. Phys. 52 (1980) 299-339. MR569166 (81e:83024) 